\title{
Modulation of certainty and valence in cardiac autonomic regulation mediated by high depressive risk
}

Hui-Ling Chan ${ }^{1 *}$, Maro G. Machizawa ${ }^{2 *}$, Ryohei Mizuochi ${ }^{3}, \&$ Shigeto Yamawaki $^{4}$

${ }^{1}$ Center for Brain, Mind and KANSEI Sciences Research, Hiroshima University, Hiroshima 7348551, Japan. Tel: +81-82-257-1722. E-mail: chanhl@hiroshima-u.ac.jp

${ }^{2}$ Center for Brain, Mind and KANSEI Sciences Research, Hiroshima University, Tokyo 130-0003, Japan. Tel: +81-50-8880-8806. E-mail: machizawa@ hiroshima-u.ac.jp

${ }^{3}$ Center for Brain, Mind and KANSEI Sciences Research, Hiroshima University, Hiroshima 7348551, Japan. Tel: +81-82-257-1722. E-mail: d145756@ hiroshima-u.ac.jp

${ }^{4}$ Center for Brain, Mind and KANSEI Sciences Research, Hiroshima University, Hiroshima 7348551, Japan. Tel: +81-82-257-1722. E-mail: yamawaki@ hiroshima-u.ac.jp

* The two authors contributed to the manuscript equally.

- Corresponding authors:

Hui-Ling Chan (chanhl@ hiroshima-u.ac.jp)

Maro G. Machizawa (machizawa@hiroshima-u.ac.jp) 


\section{Abstract}

Recent studies highlight interoception as the key to processing healthy emotion and pathophysiology of affective disorders such as major depressive disorder (MDD). It has been reported that interoceptive responses are impaired in MDD and in healthy individuals with a high depressive risk (HDR). However, it is unclear how individual differences in HDR relate to neurophysiological underpinnings for interoceptive and emotional reactions under different degrees of certainty. We examined whether HDR mediates the relationship between a neuro-physiological marker for interoception, heartbeat-evoked potential (HEP), and an index for cardiac reactivity, heart rate (HR) or heart rate variability (HRV). In a concurrent EEG-ECG experiment, 26 healthy participants underwent an emotion-evoking picture evaluation task. One of three differential auditory tones associated with a level of certainty preceded display of a pleasant or unpleasant picture. The results showed attenuated HRV activity for certain cues in HDR individuals. Neural and physiological reactions to uncertain, unpleasant pictures were enhanced by the depressive risk. These results suggest reduced responses in prediction processing and enlarged precision-weighted prediction error for unpleasant pictures. Finally, HDR significantly mediated the HEP and HR relationship for unexpected unpleasant stimuli. Our study thus provides evidence that interoceptive predictive coding in the cardiac domain is altered by HDR, and suggests that investigating the heart-brain interaction related to predictive coding will offer insights into how to estimate the level of depressive risk. 


\section{Significance Statement}

Studies have shown altered interoceptive and emotional sub-processes in major depressive disorder, as represented by neural, physiological, and behavioral disturbances. It has been speculated that alterations in depression could be due to abnormal interoceptive predictive coding. However, few studies provide direct evidence of the interrelationship among interoceptive predictive-coding, cardiac activities, and high depressive risk (HDR). The present study quantitatively provides evidence that HDR mediates the relationship between the heart and brain activity during prediction and prediction error processing in expectation and confrontation of emotional events. Our observation proposes that the quantified abnormality in autonomic responses to emotional predictive coding can be a potential neuro- and biomarker for depressive risk within the reportedly healthy population. 


\section{Introduction}

Emotion is affected by an external or internal event and is governed by the intrinsic force to maintain the current emotional state (Kuppens and Verduyn, 2017). Among various types of emotion, depression, or an emotional state marked by a constantly low mood, is characterized by alternation of emotional processing. Accumulating evidence suggests that interoception, the perception of bodily states, is a key to understanding emotional processing and the pathology of major depressive disorder (MDD) (Critchley and Garfinkel, 2017). Behavioral (Eggart et al., 2019) and neuroimaging (Avery et al., 2014; Wiebking et al., 2015) studies of MDD patients have reported impaired interoceptive functions in MDD. While neural mechanisms in depression have been extensively explored to date, particularly in regard to processing of emotion (Stuhrmann et al., 2011), the relationship between interoception and autonomic regulation has been little explored.

Our cardiac autonomic systems automatically respond to emotional events. For example, heart rate (HR) (Pollatos et al., 2007; Ilves and Surakka, 2012; Brouwer et al., 2013) and heart rate variability (HRV) (Vaschillo et al., 2008; Kop et al., 2011) are well-known markers for cardiac reactivity that reflect changes in valence (pleasant or unpleasant emotional states). Heartbeat-evoked potentials (HEPs) represent neural responses for each heartbeat and reflect interoceptive cardiac processing in the brain (Park and Blanke, 2019; Coll et al., 2021). It has been proposed that generation of HEPs may reflect projection of cardiac afferent signals from the heart to brain regions, such as the insula, where interoception is signaled from the vagus nerve and brainstem (Kern et al., 2013; Park et al., 2017; Salamone et al., 2020).

Disturbance of interceptive responses in depression may be associated with impaired predictive models or insensitivity to afferent signals (Barrett et al., 2016; Smith et al., 2021). According to the predictive coding theory of emotion (Friston and Kiebel, 2009; Seth et al., 2012), the brain constantly predicts future sensory inputs and updates the predictive model based on errors between the prediction and actual signals. As a homeostatic function to minimize (prediction) error, the body prepares and regulates its own states even before the anticipated event (Sterling, 2012). It has been proposed that uncertainty induces greater physiological (Murakami et al., 2010; Tracy et al., 2017) and neurophysiological responses (Marshall et al., 2017; Gentsch et al., 2019), especially for negative items. 
Not only persons with MDD, but also individuals with 'high depressive risk (HDR)' who have elevated risks of developing MDD (Fergusson et al., 2005) show altered interoception, such as HR recovery rate in response to emotional items (Zorzi et al., 2021) and reduced connectivity in the brain interoceptive network (Hwang et al., 2015; Peng et al., 2020). Abnormal anticipation of future events has also been linked to HDR (Argyris Stringaris et al., 2015; He et al., 2019). Thus, alteration of interoceptive responses may span the entire spectrum of depressive traits. However, it is still unclear whether interoceptive neuro-cardiac emotional responses when facing certainty and uncertainty reflect individual differences in HDRs.

The present study investigated cardiac autonomic regulation during anticipation and perception of emotional pictures in healthy individuals under uncertainty. Both electroencephalograms (EEG) and electrocardiograms (ECG) were monitored while participants performed an emotion-evoking picture evaluation task. Pre-cue tone was played to induce anticipatory responses, followed by a display of either a pleasant or unpleasant image. As an index of neuro-physiological interaction, HEP was assessed and HR and HRV were assessed as indices of cardiac reactivity. Based on previous literature on cardiac reactivity we used predictive coding theory to hypothesize that stimuli with negative valence (unpleasant pictures) under uncertainty would elicit larger cardiac responses. Moreover, we speculated that HDR would mediate the relationship between central processing of cardiac signals and cardiac reactivity indices. Altogether, we examined how cardiac and neurophysiological signals interact with interoceptive prediction and prediction errors in association with HDR.

\section{Materials and Methods}

\section{Participants}

Twenty-six healthy participants (16 females; mean and standard deviation (SD) of age: $22.19 \pm 1.86$ years old) with no history of psychiatric, psychological, or cardiac disorders participated in concurrent EEG-ECG recording during an emotion-evoking picture evaluation task. Data were acquired from a previous study (Machizawa et al., 2020). 


\section{Questionnaire procedure}

The level of HDR was evaluated using the Japanese version of Beck Depression Inventory-II (Beck et al., 1996; Spielberger et al., 2003). The overall mean and SD of the BDI score were $5.65 \pm 7.22$. For display purposes for some data analyses, participants were split into two groups. Participants having BDI scores higher than the third quartile $(B D I \geq 5)$ and lower than the first quartile $(B D I \leq 2)$ were categorized into the high depressive risk (HDR) and non-high depressive risk (non-HDR) groups, respectively. According to this criterion, 13 participants were categorized as non-HDR (BDI $=1.23 \pm 0.89)$ and 8 as $\mathrm{HDR}(\mathrm{BDI}=14.12$ $\pm 7.42)$.

\section{Behavioral task and procedure}

The emotion-evoking picture evaluation experiment consisted of 240 trials. Each trial was comprised three phases: cue, picture-presentation, and evaluation phases. During the 'cue' phase, an auditory tone was presented for $0.25 \mathrm{~s}$, followed by a 3.75-s blank period. During a picture-presentation ('pic' phase), either a pleasant or an unpleasant picture was displayed for $4 \mathrm{~s}$. Participants rated their emotional valence on a four-point Likert scale (from most unpleasant to most pleasant) after seeing a picture during the evaluation phase. Three different tones (low, mid, and high at 500,1000, and $1500 \mathrm{~Hz}$, respectively) were used as auditory pre-cues to indicate three predictive conditions: predictable pleasant (PP), predictable unpleasant (PU), and unpredictable (UX). The unpredictable condition contained two conditions based on the valence of the picture actually shown: unpredictable pleasant (UP) and unpredictable unpleasant (UU). Note that we abbreviate the conditions in this manuscript as follows: the letter $\mathrm{X}$ indicates the combination of possible conditions. For example, XP represents a combination of PP and UP conditions (pleasant picture conditions regardless of predictive tones), whereas PX represents a mixture of PU and PP conditions (predictable conditions regardless of the subsequent valence of picture). The mid $(1000-\mathrm{Hz})$ tone was the cue for the UX condition. The $500-$ and $1500-\mathrm{Hz}$ tones were used in either PP or PU conditions (the assignment was counterbalanced across participants). Each of the three predictive conditions consisted of 80 trials. In the UX condition, 40 trials showed pleasant pictures (UP) and another 40 showed unpleasant pictures (UU). Pictures were selected from the International Emotional Picture System (IAPS) (Lang et al., 1997). Finally, participants rated their emotions after seeing the images. For further details, please refer to Machizawa et al. (2020). 


\section{EEG-ECG simultaneous recordings}

During the task, brain and cardiac activity were simultaneously recorded using the BioSemi ActiveTwo system (BioSemi, Amsterdam) at a sampling rate of 2,048 Hz. The 64-channel EEG electrodes were placed according to the conventional international 10-20 montage. In addition to EEG channels, ECG electrodes were placed on the left wrist and right side of the back of the neck to monitor bipolar ECG signals. Additionally, a conventional bipolar electrooculograph (EOG) was monitored to measure vertical and horizontal eye movements. Four EOG electrodes were individually placed above and below the right eye and left and right of the outer canthi. An additional channel was placed at the tip of the nose for offline re-reference.

\section{HR and HRV computation}

Preprocessing and R peaks detection from ECG signals were conducted using the Python package, BioSPPy (Carreiras et al., 2015). Raw ECG signals were first bandpass-filtered with an FIR filter with a passband frequency range of 3-45 Hz, followed by detection (Hamilton, 2002) and location correction of $R$ peaks by relocating each $R$ peak to the time point with the maximum ECG signal within the time range of -50 to $50 \mathrm{~ms}$.

Instantaneous heart rate (HR) was computed from the inverse of the inter-beat interval (IBI between two successive R peaks. For subsequent analyses, instantaneous HR data points exceeding the physiological range (40-200 bpm) were discarded. Finally, time series of HR were obtained by applying boxcar smoothing with a window size of 3 and cubic spline interpolation to those instantaneous HR.

As an HRV index, we employed SD of normal-to-normal IBI (SDNN) in the current study (Task Force of the European Society of Cardiology the North American Society of Pacing Electrophysiology, 1996). To obtain the time series of HRV, IBIs with z-scores larger than 1.96 were discarded and then the remaining IBIS were up-sampled to $4 \mathrm{~Hz}$ using cubic spline interpolation, after which a 10 -s sliding window with a 0.25 -s step was applied and the SD of IBIs was computed within the window as the instantaneous HRV value at the center of the window. The resulting 4-Hz time series of HRV was further up-sampled to the original sampling rate of EEG at 2,048 $\mathrm{Hz}$ for accurate epoching. We set the following rejection criteria to assure the quality of HR and HRV indices. Epochs of HR and HRV were extracted between $1 \mathrm{~s}$ before and $20 \mathrm{~s}$ 
after cue display onset. For each participant, two types of outliers were detected across and within trials and discarded according to robust z-score criteria: (1) epochs with absolute robust z-scores larger than a threshold of 3.4807 (equivalent to $p=.0005$ ) after applying a robust $z$ transform to mean values of all epochs, and (2) epochs with robust z-scores of any sample larger than the threshold after applying a robust $z$ transform to the time series within the same epoch. These cleaned data were employed in subsequent analyses. The remaining epochs of HR and HRV were downsampled to $200 \mathrm{~Hz}$ and averaged across epochs of the same condition within participants for subsequent analysis.

\section{HEP computation}

EEG signals were preprocessed using EEGLAB toolbox v2019.1 for Matlab (Delorme and Makeig, 2004). Continuous raw data were bandpass-filtered (1-40 Hz), downsampled to $256 \mathrm{~Hz}$, epoched to cue-onset ($1 \mathrm{~s}$ to $10 \mathrm{~s}$ ), and baseline-corrected ( $-1 \mathrm{~s}$ to $0 \mathrm{~s})$. Artifactual epochs were detected by the automatic rejection algorithm implemented in EEGLAB. Eye-related artifacts were corrected using automatic EOG correction with conventional recursive least squares regression. An extended Infomax-independent component analysis was performed to remove EOG- and ECG-related components. Noise-free epochs were then re-referenced to the average reference of EEG signals.

For each of the EEG epochs, the time of R peaks was detected from corresponding bandpass-filtered ECG signals for two periods $0.5-3.4 \mathrm{~s}$ after cue-onset (within the cue phase) and 4.5-7.4 s after cue-onset (equivalent to $0.5-3.4 \mathrm{~s}$ after picture-onset; during the pic phase). In this study, the R peaks close to cue and picture presentations were not applied to HEP computation in order to minimize direct perceptual effects of auditory and visual stimuli, respectively.

EEG-ECG epochs containing falsely detected R peaks were then discarded based on visual inspection. For retained EEG-ECG epochs, time points of $\mathrm{R}$ peaks were used as the onset time to further epoch EEG signals ( -0.1 to $0.6 \mathrm{~s}$ ), followed by baseline correction ( -0.1 to $0 \mathrm{~s}$ ). HEPs were then computed for corresponding conditions during the cue and pic phases separately. Note that onset times of HEP signals were the time points of $\mathrm{R}$ peaks, as per convention, while that of cardiac reactivity was the time of cue display onset. 
To identify morphology of HEPs, surrogate HEPs were computed based on surrogate R-peaks that were randomly jittered in a range of -0.1 to $0.1 \mathrm{~s}$ from the time points of real $\mathrm{R}$ peaks (Gentsch et al., 2019). HEPs and surrogate HEPs of each participant were then individually averaged across all epochs regardless of condition. A nonparametric cluster-level F-test for spatiotemporal data implemented in MNE-Python (Gramfort et al., 2013) was then applied to the averaged HEP and averaged surrogate HEP waveforms to identify clusters of channels and time periods revealing significant neural responses to heartbeats within the time-window of 0.1 to $0.6 \mathrm{~s}$ after the R-peak onset. Any electrodes located within $5 \mathrm{~cm}$ of each other were considered spatial neighbors. The number of permutations was set to 2,500 and the threshold to form clusters was an F-value corresponding to an uncorrected $p$-value of .00001 .

\section{Statistical analysis}

\section{Effects of cues or emotional pictures on HR and HRV}

To identify the period showing significant main effects of cues and emotional pictures, repeatedmeasures ANOVA (RM-ANOVA) with post hoc tests on the HR or HRV was performed for each time point, followed by multiple comparison correction using the false discovery rate (FDR). As recovery duration (waveform returned to baseline) for HR and HRV appeared to differ, we considered different timewindows for HR and HRV: $12 \mathrm{~s}$ for HR waveform and $20 \mathrm{~s}$ for HRV waveform. The above-mentioned statistical procedure was conducted on HR and HRV time series for both non-HDR and HDR groups for all participants. In this study, computation of RM-ANOVA, $t$-tests, and FDR correction were performed using implementations in the Python packages, pingouin (Vallat, 2018), SciPy (Virtanen et al., 2020), and statsmodels (Seabold and Perktold, 2010), respectively. In this study, our alpha-level for FDR-corrected $p$ values ( $\left.p_{\text {FDR-corrected }}\right)$ was set at 0.05 . As necessary, uncorrected $p$-values ( $p_{\text {uncorrected }}$ ) less than 0.05 were also reported to show the trend for completeness.

\section{Effects of certainty and valence at anticipation and perception stages}

Effects of certainty on cardiac reactivity were further examined on anticipation and perception stages. Time periods of the two stages were determined based on durations showing a significant main effect of cue and valence of pictures. If no significant main effect was found during the cue phase, the whole time period $0-4 \mathrm{~s}$ during the cue phase was averaged. RM-ANOVA on averaged HR or HRV for various cues was 
performed at both stages. For each stage, the effect of certainty on cardiac reactivity was assessed between certain (PP and PU) and uncertain (UP and UU) conditions using paired $t$-tests. Moreover, the certainty induction effect was also assessed on the difference of time-averaged cardiac reactivity between the predictable and unpredictable conditions followed by the display of unpleasant pictures (PU - UU) and pleasant pictures (PP - UP), regardless of valence (PX - UX).

The effect of valence on HR and HRV was also examined for each stage. The procedure was similar to that for analyzing the effect of certainty. Durations showing a significant main effect of the picture were used to determine durations of the anticipation and perception stages. RM-ANOVA on the averaged HR or HRV for various valences at the two stages was performed. For each stage, the comparison of cardiac reactivity between pleasant (PP and $\mathrm{PU}$ ) and unpleasant ( $\mathrm{PU}$ and $\mathrm{UU}$ ) conditions was assessed using paired $t$-tests. The valence induction effect was also computed as the difference of time-averaged cardiac reactivity between the pleasant picture and unpleasant picture conditions in regard to high certainty (PP - PU), low certainty (UP - UU), and a mixture of the two (XP - XU). Effect sizes of RM-ANOVA and paired $t$-tests were measured using partial eta-squared $\left(\eta^{2}\right)$ and Hedges' $g$, respectively.

\section{Interrelation between HDR, cardiac reactivity, and HEP}

To qualify the relationship between HDRs and cardiac reactivity, the partial correlation between BDI scores and cardiac reactivity was computed for every stage and condition, using the Python package pingouin (Vallat, 2018), and was controlled for age and sex. Also, the partial correlations between BDI and the valence induction effect and between BDI and the certainty induction effect were computed. Moreover, for each index of cardiac reactivity, a comparison between non-HDR and HDR groups was performed using two-sample $t$-tests. In this study, effect sizes of two-sample $t$-tests and partial correlation were measured using Hedges' $g$ and $r^{2}$, respectively.

The raw amplitude of HEP under each condition at each phase was compared between non-HDR and HDR groups with two-sample $t$-tests, and its relationships to HDR and cardiac reactivity at the perception stage were examined using partial correlation controlled for age and sex. To reduce the bias of individual differences in EEG signal amplitude, the difference of HEP amplitude between pic and cue phases (pic- 
bioRxiv preprint doi: https://doi.org/10.1101/2021.12.06.471520; this version posted December 7, 2021. The copyright holder for this preprint (which was not certified by peer review) is the author/funder. All rights reserved. No reuse allowed without permission.

cue difference), between unpleasant and pleasant conditions (valence induction effect), and between predictable and unpredictable conditions (certainty induction effect) for each spatiotemporal cluster was also computed to examine the effect of HDR on HEP. The group difference of HEP between non-HDR and HDR groups was compared using two-sample $t$-tests. The relationship between HDR and HEP was computed with partial correlation to BDI, controlled for age and sex. Furthermore, the partial correlation between the pic - cue difference of HEP and cardiac reactivity at the perception stage was computed. The partial correlation between the valence induction effect of HEP at the cue or pic phase and the valence induction effect of cardiac reactivity at the perception stage was computed under predictable and unpredictable conditions. The partial correlation between the certainty induction effect of HEP at the cue or pic phase and the certainty induction effect of cardiac reactivity at the perception stage was computed under pleasant picture and unpleasant picture conditions.

For conditions showing a significant correlation between HR/HRV/HEP and HDR or showing a significant difference between non-HDR and HDR groups, mediation analysis implemented in pingouin (Vallat, 2018) was applied to examine the following model: the relationship between the HEP and cardiac reactivity mediated by HDR, controlled for age and sex.

\section{Results}

\section{Effects of valence, uncertainty, and HDR on HR}

HR slightly increased after cue display onset and then decreased (Figure 1a). HR time series were strongly modulated by emotional pictures from $5.4-10.7 \mathrm{~s}$ after cue presentation $\left(p_{\mathrm{FDR} \text {-corrected }}<.05\right.$ and duration $>$ $1 \mathrm{~s}$ in cue $\times$ pic RM-ANOVA for main effect of pic). During this time period, unpleasant pictures elicited a larger deceleration of HR than pleasant pictures, especially in the unpredictable condition ( $p_{\text {FDR-corrected }}$ $<.05$ and duration $>1 \mathrm{~s}$ in post hoc analysis for main effect of pic). Modulation of HR time series by emotional pictures also occurred in the HDR group at 4.2-7.1 s and 8.5-11.9 s (Figure 1c), but not in the non-HDR group (Figure 1b). The HR time series were also modulated by cue at the cue phase (1.7-3.0 s) and the pic phase $(8.2-9.7 \mathrm{~s})\left(p_{\text {uncorrected }}<0.05\right.$ and duration $>1 \mathrm{~s}$ in cue $\times$ pic RM-ANOVA for main effect of cue). Greater acceleration of HR at 1.7-3.0 s (UX > PP) and smaller deceleration at 8.2-9.7 s (UP < PP 
and $\mathrm{UX}<\mathrm{PU}$ ) appeared in the unpredictable condition than the predictable condition. Modulation of $\mathrm{HR}$ time series by cue was also observed at $0.8-4.7 \mathrm{~s}$ and $7.7-12.0 \mathrm{~s}$ in the non-HDR group, but not in the HDR group ( $p_{\text {uncorrected }}<.05$ and duration $>1 \mathrm{~s}$ ). Moreover, both the cue and pic modulated HR time series at 2.3-3.5 s ( $p_{\text {uncorrected }}<0.05$ and duration $>1 \mathrm{~s}$ in cue $\times$ pic RM-ANOVA for interaction of cue and pic). HR was significantly different between anticipation stage (1.7-3.0 s) and perception stage (8.2-9.7 s) in the unpleasant picture conditions (PU and UU, $\mathrm{F}=26.04, p<.001, \eta^{2}=.51$, in stage $\times$ cue RM-ANOVA for main effect of stage) and also the pleasant picture conditions (PP and UP, F = 20.19, $p<.001, \eta^{2}=.45$ ) (Figure 2a). Significantly reduction of HR from anticipation stage to perception stage was found in the unpleasant condition $(t=7.09, p<.001$, Hedges' $g=1.64$, in post hoc analysis for the main effect of stage) and pleasant condition ( $t=6.18, p<.001$, Hedges' $g=1.36)$. HR was significantly different between certain and uncertain cue conditions for pleasant picture condition $\left(F=7.14, p=.013, \eta^{2}=.22\right.$ in stage $\times$ cue RM-ANOVA for main effect of cue), but not for the unpleasant picture condition ( $F=0.06, p=.813, \eta^{2}$ $<.01)$. In the pleasant picture condition, unpredictive cues induced higher HR than predictive cues $(\mathrm{PP}<$ UP, $t=6.18, p<.001$, hedges' $g=1.36$ in post hoc analysis for the main effect of cue) and the difference of HR responses to the two types of cues was prominent at the anticipation stage $\left(t=2.63, p_{\text {uncorrected }}\right.$ $=.014, p_{\text {FDR-corrected }}=.021$, Hedges' $g=.26$, in paired $t$-test) .

A significant difference in HR responses between the anticipation stage (0-4.0 s) and the perception stage (5.4-10.7 s) was also found in the predictive cue condition ( $P U$ and $P P, F=29.86, p<.001, \eta^{2}=.54$, in stage $\times$ valence RM-ANOVA for main effect of stage) and the unpredictive cue condition (UU and UP, $F$ $=33.22, p<.001, \eta^{2}=.57$ ) (Figure $2 \mathrm{~b}$ ). HR reduced from the anticipation stage to the perception stage in the predictive cue condition $(t=7.44, p<.001$, Hedges' $g=1.57$, in post hoc analysis for the main effect of stage) and unpredictive cue condition ( $t=7.79, p<.001$, Hedges' $g=1.63)$. HR responses were also strongly modulated by valence $\left(\mathrm{F}=11.64, p=.002, \eta^{2}=.32\right.$, in stage $\times$ valence RM-ANOVA for main effect of valence). Higher HR was observed in the pleasant picture condition than the unpleasant picture condition (UP $>\mathrm{UU}, t=7.79, p<.001$, Hedges' $g=1.63$, in post hoc analysis for main effect of valence) and the difference of $\mathrm{HR}$ responses to the two types of pictures was prominent at the perception stage ( $t$ $=3.45, p_{\text {uncorrected }}=.002, p_{\text {FDR-corrected }}=.006$, Hedges' $g=.72$, in paired $t$-test) . 
bioRxiv preprint doi: https://doi.org/10.1101/2021.12.06.471520; this version posted December 7, 2021. The copyright holder for this preprint (which was not certified by peer review) is the author/funder. All rights reserved. No reuse allowed without permission.

At the anticipation stage, HR responses were not altered with the HDR (in two-sample $t$-test for non-HDR and HDR groups and in partial correlation analysis between BDI and anticipatory HR). At the perception stage (5.4-10.7 s), HR changes in the UU and XU conditions were significantly correlated with BDI scores (Figure 2c and Table 1). Moreover, the induction valence effect under the unpredictable condition (UP UU) was also significantly correlated with BDI scores $\left(r=.43, p=.036, r^{2}=.185\right)$. Furthermore, unpleasant pictures significantly induced larger deceleration of HR in the HDR group than in the non-HDR group ( $t=$ 5.41, $p_{\text {uncorrected }}<.001$, Hedges $g=2.08$ for UU and $t=3.86, p_{\text {uncorrected }}<.001$, Hedges' $g=1.52$ for XU in two-sample $t$-tests).

\section{Effect of valence, uncertainty and HDR on HRV}

HRV increased after cue display onset, exceeded the maximum peak at around $10 \mathrm{~s}$, and then recovered at around $20 \mathrm{~s}$ (Figure 3a). HRV time series were strongly modulated by emotional pictures at $14.8-16.1 \mathrm{~s}$ ( $p_{\text {FDR-corrected }}<.05$ and duration $>1 \mathrm{~s}$ in cue $\times$ pic RM-ANOVA for main effect of pic). Modulation of HRV time series was also observed in the non-HDR group at 10.0-15.8 s $\left(p_{\mathrm{FDR}-\text { corrected }}<.05\right.$ and duration $>1 \mathrm{~s}$ in cue $\times$ pic RM-ANOVA for main effect of pic), but not in the HDR group. In the non-HDR group, modulation of HRV time series by emotional pictures was prominent in unpredictive conditions, UP > UU, at 9.9-16.0 $\mathrm{s}\left(p_{\text {FDR-corrected }}<.05\right.$ and duration $>1 \mathrm{~s}$ in post hoc analysis for main effect of pic).

HRV time series were not significantly modulated by cue $\left(p_{\text {uncorrected }}<.05\right.$ and duration $>1 \mathrm{~s}$ in cue $\times$ pic RM-ANOVA for main effect of cue). The interaction of cue and emotional picture was also not observed. In the non-HDR group, HRV time series were modulated by cue during $2.9-5.7 \mathrm{~s}$ (Figure $3 \mathrm{~b}$ ) while modulation of HRV time series by cue was observed in the HDR group at 1.5-3.2 s and 9.2-12.1 s ( $p_{\text {uncorrected }}<.05$, duration $>1 \mathrm{~s}$ in cue $\times$ pic RM-ANOVA for main effect of cue) (Figure 3c). HRV differed significantly between predictable and unpredictable conditions at the cue phase, but the trend in the non-HDR group (PU $>$ UX at 2.9-5.7 s, $p_{\text {uncorrected }}<.05$, duration $>1 \mathrm{~s}$ in post hoc analysis) and that in the HDR group (PU $<U U$ and $P U<U X$ at 1.5-3.2 s) were reversed.

By combining time periods showing significant main effects of cue in the non-HDR and HDR groups, 1.5$4.0 \mathrm{~s}$ and $9.2-12.1 \mathrm{~s}$ were selected as the anticipation and perception stages to further examine effects of cue and stage. The HRV was strongly modulated by stage in both unpleasant picture (PU and UU, F = 7.63, 
$p=.011, \eta^{2}=.23$ in stage $\times$ cue RM-ANOVA for the main effect of stage) and pleasant picture (PP and UP, $\mathrm{F}=23.38, p<.001, \eta^{2}=.48$ ) conditions. In the pleasant picture condition, the HRV was strongly modulated by cue $\left(F=5.62, p=.026, \eta^{2}=.18\right.$ in stage $\times$ cue RM-ANOVA for the main effect of cue $)$ and the interaction of cue and stage $\left(\mathrm{F}=9.02, p=.006, \eta^{2}=.27\right.$ in stage $\times$ cue RM-ANOVA $)$ was also observed.

Based on the results of main effect of picture from all participants, $0-4.0 \mathrm{~s}$ and $10.0-15.8 \mathrm{~s}$ were selected as the anticipation and perception stages for further examination of the effects of valence and stage. The HRV was strongly modulated by stage in both predictive cue (PU and PP, F =9.61, $p=.004, \eta^{2}=.28$ in stage $\times$ cue RM-ANOVA for the main effect of stage) and unpredictive cue (UU and UP, $F=12.44, p$ $\left.=.002, \eta^{2}=.33\right)$ conditions. The interaction of cue and stage was found in the unpredictive cue condition $\left(\mathrm{F}=7.79, p=.010, \eta^{2}=.24\right.$ in stage $\times$ cue RM-ANOVA $)$. At the perception stage, UP pictures elicited larger HRV increases than did PP pictures (9.2-12.1 s, $t=2.87, p_{\text {uncorrected }}=.008, p_{\text {FDR-corrected }}=.012$, Hedges' $g$ $=.53$ in paired $t$-test $)$ and UU pictures $\left(10.0-15.8 \mathrm{~s}, t=2.46, p_{\text {uncorrected }}=.021, p_{\text {FDR-corrected }}=.032\right.$, Hedges' $g=.53$ ) (Figures $4 a$ and $4 b$, respectively).

At the anticipation stage (1.5-4.0 s), reduced HRV responses to either pleasant or unpleasant stimuli under predictable conditions were correlated with HDR (Figure 4c). Under unpredictable conditions, anticipatory HRV responses were not correlated with BDI scores (Figure 4c). Moreover, compared to the non-HDR group, the HDR group showed significantly low anticipatory HRV responses in PU conditions ( $t=$ 4.37, $p_{\text {uncorrected }}=.001$, Hedges $g=1.53$ in two-sample $t$-tests) and a reversed certainty induction effect under unpleasant picture conditions, $\mathrm{PU}-\mathrm{UU}\left(t=2.92, p_{\text {uncorrected }}=.010\right.$, Hedges' $\left.g=1.04\right)$ (Supplementary Table 1). Thus, anticipatory HRV responses in predictable conditions were altered with the level of HDR.

At the perception stage of 9.2-12.1 s, the certainty induction effect on the processing of unpleasant pictures, $\mathrm{PU}-\mathrm{UU}$, was significantly stronger in the HDR group than in the non-HDR group $(t=2.36$, $p_{\text {uncorrected }}=.003$, Hedges' $g=1.35$ in two-sample $t$-test) and significantly correlated with BDI scores $(r=$ $-.49, p=.016, r^{2}=.238$ in partial correlation analysis) (Table 2). At the perception stage of 10.0-15.8 $\mathrm{s}$, 
the valence induction effect in the unpredictable condition (UP - UU) was larger in the non-HDR group than the HDR group $\left(t=3.37, p_{\text {uncorrected }}=.003\right.$, Hedges' $g=1.36$ ) and was negatively correlated with HDR $\left(r=-.60, p=.002, r^{2}=.359\right)$ (Supplementary Table 2). Moreover, HRV changes in the UU condition averaged over 9.2-12.1 s (Table 2) and over 10.0-15.8 s (Supplementary Table 2) were both significantly higher in the HDR group than in the non-HDR group and positively correlated with the level of HDR. The HRV in the UU condition in the HDR group was relatively higher than in the other three conditions (Figure 3c), but that in the non-HDR group was relatively lower than that in the other three conditions (Figure 3b). Thus, perceptual HRV responses in the UU condition were largely altered with the level of HDR.

\section{Spatiotemporal clusters of HEP}

Comparing the grand averages of the original HEP and surrogate HEP time series, the original one appeared to evoke stronger signals than the surrogate one (Figure 5a). In the nonparametric cluster-level test over original HEP and surrogate HEP spatiotemporal data $(p<.05$, duration $>0.1 \mathrm{~s}$, number of channels >= 3), three significant spatiotemporal clusters were observed over (1) the occipital and left temporoparietal regions during $0.10-0.40 \mathrm{~s},(2)$ the central region during $0.10-0.29 \mathrm{~s}$, and (3) the right temporal regions during $0.23-0.37 \mathrm{~s}$ (Figure $5 \mathrm{~b}$ ). Cluster 1 showed a positive deflection, while clusters 2 and 3 showed negative deflections.

\section{Effect of HDR on HEP cluster}

The mean amplitude of the HEP cluster was non-significant between the non-HDR and HDR groups (Figure 6a). However, the partial correlation analysis demonstrated that weak amplitude of HEP clusters 1 and 3 correlated with the HDR when processing unpredictive cues (UX condition) (Supplementary Table 3). Furthermore, compared to the non-HDR group, the HDR group revealed a significantly larger pic - cue difference in HEP cluster 3 under the UU condition $\left(t=3.19, p_{\text {FDR-corrected }}=.033\right.$, Hedges' $g=1.49$ in twosample $t$-test) (Figure 6b). In the HDR group, the negative component of HEP in the UU condition increased at the pic phase compared to the cue phase (Figure 7d). In contrast, the HEP of the non-HDR group in the UU condition decreased at the pic phase. The results of partial correlation analysis further support that the pic - cue difference of cluster 3 enlarged as the HDR increased (Table 3). 
In the unpleasant picture condition, the certainty induction effect found in cluster 3 at the pic phase was significantly larger in the HDR group than in the non-HDR group (PU $-U U, t=3.71, p_{\text {FDR-corrected }}=.006$, Hedges' $g=1.46$ ) (Figure 6c). Under unpleasant picture conditions, unpredictable pictures elicited greater HEP amplitude than predictable pictures in the HDR group, while HEP amplitude elicited by the two types of pictures was similar in the non-HDR group (Figure 7b and 7d). However, significant partial correlation between the certainty induction effect and the HDR was only found in cluster 1 at cue phase under unpleasant picture condition (PU - UU, $r=.45, p_{\text {uncorrected }}=.038$ ) (Supplementary Table 4).

In the predictable condition, the valence induction effect found in cluster 3 at the cue phase was significantly larger in the HDR group than in the non-HDR group (PU $-\mathrm{PP}, t=3.10, p_{\mathrm{FDR} \text {-corrected }}=.034$, Hedges' $g=1.41)$ (Figure 6d). Unpleasant stimuli also elicited greater HEP amplitude than pleasant stimuli in the HDR group, while unpleasant stimuli elicited lesser HEP amplitude than pleasant stimuli in the nonHDR group (Figure 8). However, no significant partial correlation was found between the valence induction effect and the HDR (Supplementary Table 5).

\section{Correlation between HEP cluster and cardiac reactivity}

In the UU condition, the mean amplitude of HEP clusters 1 and 2 at pic phase was correlated with HR changes at the pic phase (5.4-10.7 s) (Table 4) and that of HEP cluster 3 at cue phase was significantly correlated with HRV changes at the pic phase (9.2-12.2 s) (Supplementary Table 6). In the PP condition, pic - cue differences in HEP cluster 3 were negatively correlated with the change of HRV at the pic phase (Supplementary Table 7). This indicated that the larger negativity of HEP amplitude in cluster 3 at the pic phase was associated with larger HRV responses under the PP condition.

In the PP - UP condition, certainty induction effects on the HEP cluster 1 at the pic phase and cue phase were negatively correlated with the certainty induction effect on HR and HRV changes, respectively (Supplementary Table 8). This indicates that in the pleasant picture condition, a larger certainty induction effect on the HEP amplitude of cluster 1 at the pic and cue phase caused a smaller certainty induction effect on the HR and HRV changes, respectively. In the PU - PP condition, valence induction effects on the HEP cluster 3 at the pic phase and on the cluster 1 at the cue phase were negatively correlated with the valence induction effect on HR changes and HRV changes, respectively (Supplementary Table 9). In 
other words, in the predictable condition, larger valence induction effects on the HEP amplitude of cluster 1 at the cue phase and cluster 3 at the pic phase caused larger valence induction effects on the $H R V$ and $H R$, respectively.

\section{Mediation analysis}

Table 1 and 2 show that HR and HRV responses in the UU condition at the perception stage reflected HDRs, respectively. The mean HEP amplitude at cue phase under the UX condition and the pic - cue difference under the UU conditions in cluster 3 were altered with HDR (Supplementary Table 3 and Table 3). In the UU condition, the mean HEP amplitude at cue phase showed a tendency to predict cardiac reactivity at pic phase (Table 4 and Supplementary Table 6). Thus, we further examined the mediation effect of HDR on the relationship between HEP of cluster 3 and cardiac reactivity in the UU condition. This analysis showed that the indirect effect of mean HEP amplitude on HR changes mediated by HDR was significant, whereas the direct effect was non-significant (Figure 9a). Moreover, the indirect effect of pic cue HEP amplitude difference on HR changes mediated by HDR was significant, whereas the direct effect was non-significant (Figure 9b). On the other hand, indirect and direct effects of HEP cluster 3 on HRV changes mediated by HDR were both non-significant when using pic - cue HEP amplitude difference or mean amplitude of HEP. This suggests that the HDR fully explained the association between HEP responses and HR changes, but not HRV changes, under the UU condition.

\section{Discussion}

This study examined the extent to which HDR affects cardiac and neurophysiological emotional responses under various certainty. A comprehensive temporal characterization of $\mathrm{HR}, \mathrm{HRV}$, and HEP responses revealed dissociable and unique relations between certainty of predictive cues and emotional valence of pictures in the HDR and non-HDR groups. These results support the hypothesis that HDR alters processes for interoceptive predictive coding and increases the fluctuation of physiological responses against unpredictable stimuli. It appears that HDR influences anticipatory HRV and HEP responses and mediates the relationship between the HEP and HR responding to UU pictures. These results indicate that HDR reduces neural resources for prediction processing and causes large prediction errors against 
unpredictable negative events. The present study offers evidence for the disturbance of interoception as an indicator of HDR.

Here, the comparison of anticipatory HRV (Figures 3b-c and 4c) between the HDR and non-HDR groups revealed different effects of cues on physiological activity, consistent with the previous claim that depression is associated with dysfunction in allostatic regulation (Barrett et al., 2016). The SDNN is thought to reflect the activity of parasympathetic (PNS) and sympathetic nervous systems (SNS)(Shaffer and Ginsberg, 2017). Moreover, low resting HRV has been associated with high emotional regulation difficulty and HDR (Williams et al., 2015; Fantini-Hauwel et al., 2020). Thus, attenuated anticipatory HRV implies low cognitive flexibility (Gillie et al., 2015; Colzato et al., 2018) and autonomic capacity to regulate emotion in the HDR group. Allostasis involves prediction of body state responding to future events and preparation of the body before actual needs arise. According to Embodied Predictive Interoception Coding model (Barrett and Simmons, 2015), allostatic and anticipatory responses are modulated by interoceptive predictions arising from the anterior insula (aINS), middle/anterior cingulate cortex (MCC/ACC), and ventromedial prefrontal cortex (VmPFC). Among them, the alNS and ACC activity is altered in both subclinical depression and MDD (Hwang et al., 2015; Besteher et al., 2020). The vmPFC, INS, and MCC activity also covaries with SDNN (Schumann et al., 2021). Thus, increased anticipatory HRV in the non-HDR group enables preparation of physiological status for upcoming stimuli. Attenuated anticipatory HRV may indicate inefficient allostatic regulation in the HDR group.

Moreover, the HDR group showed enhanced frontotemporal HEPs during anticipation of unpleasant pictures compared to pleasant pictures (Figures $6 \mathrm{~d}$ and 8), suggesting an interoceptive response bias for negative valence. It is consistent with previous findings that HDR alters anticipatory responses in the INS, ventrolateral PFC, ACC, and ventral striatum (Argyris Stringaris et al., 2015; He et al., 2019). The INS, one of major neural sources underlying HEP (Park et al., 2017), is responsible for processing prediction and prediction error of current and future physiological status (Tracy et al., 2017; Livneh et al., 2020). Thus, our findings provide additional neurophysiological evidence for alterations associated with HDR in interoception. 
We observed HEP changes before (cue-phase) and after (pic-phase) the onset of target stimuli. The HDR group showed largely increasing frontotemporal HEPs from the cue-phase to pic-phase (Figures $6 \mathrm{~b}$ and 7d), implying enhanced neural processing of prediction error in frontotemporal channels. The HDR group also showed large certainty induction effects on frontotemporal HEPs responding to unpleasant pictures (Figure 6c). Consistent with previous studies, uncertain stimuli enhanced HEP amplitude (Marshall et al., 2017; Gentsch et al., 2019). HEPs reflect the precision-weighted prediction error for each individual heartbeat in the heartbeat counting task (Banellis and Cruse, 2020). Precision, or the inverse of certainty, is made based on the reliability of environment and modulated by attention (Park and Blanke, 2019; Petzschner et al., 2019; Coll et al., 2021). When updating the prediction model, precision is considered to weight prediction errors and modulates the inference (Seth and Friston, 2016; Haarsma et al., 2021). Attentional bias for negative events might be notable characteristics of both MDD and subclinical depression (Lin et al., 2019). Thus, our findings of altered HEP in HDR individuals may highlight their negative bias in predictive coding processing.

In the previous study, HDR was associated with enhanced cardiac reactivity when processing negative events (De Zorzi et al., 2021), which was also found in the UU condition in our study (Figures 1c, 2c and 3c). To understand central processing of cardiac signals, we also analyzed HEP. Compared to non-HDR, frontotemporal HEPs of HDR individuals showed larger pic-cue differences and induction effects on certainty (Figures $6 b-c$ and $7 d$ ). The observed effect on frontotemporal HEPs and HR may be interpreted in three ways. First, frontotemporal HEPs reflect the precision weighting process and the HDR group overestimates weights for prediction errors elicited by UU pictures, highlighting their negative attentional bias (Gotlib, 1983). Second, low interoceptive sensibility of visceral afferent signals during the cue phase enlarges the prediction error. MDD patients show reduced sensitivity in heartbeat perception due to low HEP amplitude (Terhaar et al., 2012) and low precision to cardiac afferent signals (Smith et al., 2020). Third, increased neural responses for images may be linked to enhanced autonomic control of cardiac dynamics, in subclinical depression during resting states (Catrambone et al., 2021). Moreover, the relationship between frontotemporal HEPs and HR was mediated by the BDI score (Figures 9a-b), leading to our conclusion that HDR plays a crucial role on the central processing of cardiac signals and cardiac 
reactivity. Altogether, our findings suggest that HDR may link to altered regulation of neurocardiac interoception against unexpected negative events, representing hypersensitivity of interoceptive prediction error that may highlight their lowered resilience against stressors (Southwick et al., 2005).

Additionally, we demonstrate temporal characteristics of HR changes during anticipation and perception of emotional pictures, the main effect of cues on HR initiated from $1.7 \mathrm{~s}$ and differential deceleration of HR between emotional pictures initiated from $5.6 \mathrm{~s}$, followed by the recovery of HR after 10 to $12 \mathrm{~s}$ (Figure 1). Activation of PNS decreases HR and activation of SNS increases HR. The PNS exerts its effect on cardiac signals after $1 \mathrm{~s}$, while the SNS exerts its effect after $5 \mathrm{~s}$ (Shaffer and Ginsberg, 2017). Thus, HR deceleration initiated in the cue phase may be modulated mainly by PNS activity and the recovery of HR reflects the effect of the SNS. Our post hoc analysis further confirmed earlier findings that pictures of negative valence decelerated HR more than those of positive valence (Pollatos et al., 2007; Bradley et al., 2008; Ilves and Surakka, 2012; Brouwer et al., 2013). However, our findings were not consistent with previous reports that unpredictable stimuli decelerated HR more than predictable ones (Murakami et al., 2010; Tracy et al., 2017). This may result from different durations for baseline correction. Previous studies commonly used the period before the presentation of pictures as baseline. Since anticipation induces changes of physiological activity (Pastor et al., 2014; Pulopulos et al., 2018), our design may be more sensitive to effects of anticipation. Together with our findings of HRV, we provide evidence that modulation of cardiac activity initiates during the anticipation stage, suggesting that research on physiological activity should consider effects of anticipation if various predictive cues are applied.

Despite our comprehensive analyses, this study has several limitations. First, since it was not feasible for our event-related paradigm and it has been suggested that accurate measures of SDNN can be obtained from 10-s ECG recordings (Munoz et al., 2015; van den Berg et al., 2018), we computed temporal dynamics of HRV using a 10-s sliding window. Our analysis of HRV may not be directly comparable to the standard short-term HRV from 5-min ECG recordings (Task Force of the European Society of Cardiology the North American Society of Pacing Electrophysiology, 1996). Second, our analysis of cardiac reactivity was limited to two time-domain parameters, HR and SDNN. As cardiac reactivity markers, frequencydomain parameters, such as low or high frequency components, might be possible. However, ECG 
bioRxiv preprint doi: https://doi.org/10.1101/2021.12.06.471520; this version posted December 7, 2021. The copyright holder for this preprint (which was not certified by peer review) is the author/funder. All rights reserved. No reuse allowed without permission.

recordings of at least 60 s are necessary (Munoz et al., 2015), so future studies could assess this using different experimental designs, such as a blocked design rather than the event-related design we employed here. Third, although we tried to dissociate the event-related effects of cue and picture on cardiac reactivity, temporal dissociation might not be clearly achievable because of the fixed time interval between cues and pictures in our paradigm. Further investigations would be required to disentangle the temporal characterization of these effects. Although limitations and discrepancies exit in this study, our evidence supports the notion that HDR affects interoceptive cardiac processing responding to cues and emotional pictures.

To conclude, our findings reveal that HDR was associated with attenuated HRV and enhanced HEP during anticipation, and that the negative bias of HDR can be monitored by enhanced cardiac reactivity and HEP for unexpected negative stimuli. Moreover, HDR was the mediator of neurophysiological responses for unexpected negative events. Altogether, neurophysiological processing of interoceptive prediction and prediction error under uncertainty and valence may be critical in HDR.

\section{Acknowledgements}

This study was supported by the Japan Science and Technology Agency from the Center of Innovation (COI) Program (Grant No. JPMJCE1311) and the Grant-in-Aid for Early-Career Scientists (Grant No. 20299115). We thank Noriaki Kanayama, at the National Institute of Advanced Industrial Science and Technology (AIST), Japan and Kai Makita, at the University of Fukui, Japan, for data collection. CHL designed research, analyzed data and helped write the paper. MGM designed research and helped write the paper. RM analyzed data. SY obtained the research grant from the COI program, supervised research, and helped write the paper.

\section{References}

Argyris Stringaris et al. (2015) The Brain's Response to Reward Anticipation and Depression in Adolescence: Dimensionality, Specificity, and Longitudinal Predictions in a Community-Based Sample. American Journal of Psychiatry 172:1215-1223.

Avery JA, Drevets WC, Moseman SE, Bodurka J, Barcalow JC, Simmons WK (2014) Major Depressive Disorder Is Associated With Abnormal Interoceptive Activity and Functional Connectivity in the Insula. Biological Psychiatry 76:258-266. 
bioRxiv preprint doi: https://doi.org/10.1101/2021.12.06.471520; this version posted December 7, 2021. The copyright holder for this preprint (which was not certified by peer review) is the author/funder. All rights reserved. No reuse allowed without permission.

Banellis L, Cruse D (2020) Skipping a Beat: Heartbeat-Evoked Potentials Reflect Predictions during Interoceptive-Exteroceptive Integration. Cerebral Cortex Communications 1:tgaa060.

Barrett LF, Simmons WK (2015) Interoceptive predictions in the brain. Nat Rev Neurosci 16:419-429.

Barrett LF, Quigley KS, Hamilton P (2016) An active inference theory of allostasis and interoception in depression. Philosophical Transactions of the Royal Society B: Biological Sciences 371:20160011.

Beck AT, Steer RA, Brown G (1996) Beck depression inventory-II. Psychological Assessment.

Besteher B, Gaser C, Nenadić I (2020) Brain Structure and Subclinical Symptoms: A Dimensional Perspective of Psychopathology in the Depression and Anxiety Spectrum. Neuropsychobiology 79:270-283.

Bradley MM, Miccoli L, Escrig MA, Lang PJ (2008) The pupil as a measure of emotional arousal and autonomic activation. Psychophysiology 45:602-607.

Brouwer A-M, van Wouwe N, Mühl C, van Erp J, Toet A (2013) Perceiving blocks of emotional pictures and sounds: effects on physiological variables. Frontiers in human neuroscience 7:295-295.

Carreiras C, Alves AP, Lourenço A, Canento F, Silva H, Fred A (2015) BioSPPy - Biosignal Processing in Python. In.

Catrambone V, Messerotti Benvenuti S, Gentili C, Valenza G (2021) Intensification of functional neural control on heartbeat dynamics in subclinical depression. Translational Psychiatry 11:221.

Coll M-P, Hobson H, Bird G, Murphy J (2021) Systematic review and meta-analysis of the relationship between the heartbeat-evoked potential and interoception. Neuroscience \& Biobehavioral Reviews 122:190200.

Colzato LS, Jongkees BJ, de Wit M, van der Molen MJW, Steenbergen L (2018) Variable heart rate and a flexible mind: Higher resting-state heart rate variability predicts better task-switching. Cognitive, Affective, \& Behavioral Neuroscience 18:730-738.

Critchley HD, Garfinkel SN (2017) Interoception and emotion. Current Opinion in Psychology 17:7-14.

Delorme A, Makeig S (2004) EEGLAB: an open source toolbox for analysis of single-trial EEG dynamics including independent component analysis. Journal of Neuroscience Methods 134:9-21.

Eggart M, Lange A, Binser MJ, Queri S, Müller-Oerlinghausen B (2019) Major Depressive Disorder Is Associated with Impaired Interoceptive Accuracy: A Systematic Review. Brain Sciences 9:131.

Fantini-Hauwel C, Batselé E, Gois C, Noel X (2020) Emotion Regulation Difficulties Are Not Always Associated With Negative Outcomes on Women: The Buffer Effect of HRV. Frontiers in Psychology 11:697.

Fergusson DM, Horwood L, Ridder EM, Beautrais AL (2005) Subthreshold Depression in Adolescence and Mental Health Outcomes in Adulthood. Archives of General Psychiatry 62:66-72.

Friston K, Kiebel S (2009) Predictive coding under the free-energy principle. Philosophical transactions of the Royal Society of London Series B, Biological sciences 364:1211-1221.

Gentsch A, Sel A, Marshall AC, Schütz-Bosbach S (2019) Affective interoceptive inference: Evidence from heartbeat evoked brain potentials. Human Brain Mapping 40:20-33.

Gillie BL, Vasey MW, Thayer JF (2015) Individual differences in resting heart rate variability moderate thought suppression success. Psychophysiology 52:1149-1160.

Gotlib IH (1983) Perception and recall of interpersonal feedback: Negative bias in depression. Cognitive Therapy and Research 7:399-412.

Gramfort A, Luessi M, Larson E, Engemann D, Strohmeier D, Brodbeck C, Goj R, Jas M, Brooks T, Parkkonen L, Hämäläinen M (2013) MEG and EEG data analysis with MNE-Python. Frontiers in Neuroscience 7:267.

Haarsma J, Fletcher PC, Griffin JD, Taverne HJ, Ziauddeen H, Spencer TJ, Miller C, Katthagen T, Goodyer I, Diederen KMJ, Murray GK (2021) Precision weighting of cortical unsigned prediction error signals benefits learning, is mediated by dopamine, and is impaired in psychosis. Molecular Psychiatry 26:5320-5333.

Hamilton P (2002) Open source ECG analysis. In: Computers in Cardiology, pp 101-104.

He Z, Zhang D, Muhlert N, Elliott R (2019) Neural substrates for anticipation and consumption of social and monetary incentives in depression. Social Cognitive and Affective Neuroscience 14:815-826.

Hwang JW, Egorova N, Yang XQ, Zhang WY, Chen J, Yang XY, Hu LJ, Sun S, Tu Y, Kong J (2015) Subthreshold depression is associated with impaired resting-state functional connectivity of the cognitive control network. Translational Psychiatry 5:e683.

Ilves M, Surakka V (2012) Heart Rate Responses to Synthesized Affective Spoken Words. Advances in HumanComputer Interaction 2012:158487.

Kern M, Aertsen A, Schulze-Bonhage A, Ball T (2013) Heart cycle-related effects on event-related potentials, spectral power changes, and connectivity patterns in the human ECoG. Neurolmage 81:178-190. 
bioRxiv preprint doi: https://doi.org/10.1101/2021.12.06.471520; this version posted December 7, 2021. The copyright holder for this preprint (which was not certified by peer review) is the author/funder. All rights reserved. No reuse allowed without permission.

Kop WJ, Synowski SJ, Newell ME, Schmidt LA, Waldstein SR, Fox NA (2011) Autonomic nervous system reactivity to positive and negative mood induction: The role of acute psychological responses and frontal electrocortical activity. Biological Psychology 86:230-238.

Kuppens P, Verduyn P (2017) Emotion dynamics. Current Opinion in Psychology 17:22-26.

Lang PJ, Bradley MM, Cuthbert BN (1997) International affective picture system (IAPS): Technical manual and affective ratings. 1:39-58.

Lin X-X, Sun Y-B, Wang Y-Z, Fan L, Wang X, Wang N, Luo F, Wang J-Y (2019) Ambiguity Processing Bias Induced by Depressed Mood Is Associated with Diminished Pleasantness. Scientific Reports 9:18726.

Livneh Y, Sugden AU, Madara JC, Essner RA, Flores VI, Sugden LA, Resch JM, Lowell BB, Andermann ML (2020) Estimation of Current and Future Physiological States in Insular Cortex. Neuron 105:1094-1111.e1010.

Machizawa MG, Lisi G, Kanayama N, Mizuochi R, Makita K, Sasaoka T, Yamawaki S (2020) Quantification of anticipation of excitement with a three-axial model of emotion with EEG. Journal of Neural Engineering 17:036011.

Marshall AC, Gentsch A, Jelinčić V, Schütz-Bosbach S (2017) Exteroceptive expectations modulate interoceptive processing: repetition-suppression effects for visual and heartbeat evoked potentials. Scientific Reports 7:16525.

Munoz ML, van Roon A, Riese H, Thio C, Oostenbroek E, Westrik I, de Geus EJC, Gansevoort R, Lefrandt J, Nolte IM, Snieder H (2015) Validity of (Ultra-)Short Recordings for Heart Rate Variability Measurements. PLOS ONE 10:e0138921.

Murakami H, Matsunaga M, Ohira H (2010) Phasic heart rate responses for anticipated threat situations. International Journal of Psychophysiology 77:21-25.

Park H-D, Blanke O (2019) Heartbeat-evoked cortical responses: Underlying mechanisms, functional roles, and methodological considerations. Neurolmage 197:502-511.

Park H-D, Bernasconi F, Salomon R, Tallon-Baudry C, Spinelli L, Seeck M, Schaller K, Blanke O (2017) Neural Sources and Underlying Mechanisms of Neural Responses to Heartbeats, and their Role in Bodily Selfconsciousness: An Intracranial EEG Study. Cerebral Cortex 28:2351-2364.

Pastor MC, Poy R, Segarra P, Moltó J (2014) Emotional Reactivity during Anticipation and Perception of Affective Pictures. The Spanish Journal of Psychology 17:E110.

Peng X, Lau WKW, Wang C, Ning L, Zhang R (2020) Impaired left amygdala resting state functional connectivity in subthreshold depression individuals. Scientific Reports 10:17207.

Petzschner FH, Weber LA, Wellstein KV, Paolini G, Do CT, Stephan KE (2019) Focus of attention modulates the heartbeat evoked potential. Neurolmage 186:595-606.

Pollatos O, Herbert BM, Matthias E, Schandry R (2007) Heart rate response after emotional picture presentation is modulated by interoceptive awareness. International Journal of Psychophysiology 63:117-124.

Pulopulos MM, Vanderhasselt M-A, De Raedt R (2018) Association between changes in heart rate variability during the anticipation of a stressful situation and the stress-induced cortisol response. Psychoneuroendocrinology 94:63-71.

Salamone PC, Sedeño L, Legaz A, Bekinschtein T, Martorell M, Adolfi F, Fraile-Vazquez M, Rodríguez Arriagada N, Favaloro L, Peradejordi M, Absi DO, García AM, Favaloro R, Ibáñez A (2020) Dynamic neurocognitive changes in interoception after heart transplant. Brain Communications 2:fcaa095.

Schumann A, de la Cruz F, Köhler S, Brotte L, Bär K-J (2021) The Influence of Heart Rate Variability Biofeedback on Cardiac Regulation and Functional Brain Connectivity. Frontiers in Neuroscience 15:775.

Seabold S, Perktold J (2010) statsmodels: Econometric and statistical modeling with python. In: 9th Python in Science Conference.

Seth A, Suzuki K, Critchley H (2012) An Interoceptive Predictive Coding Model of Conscious Presence. Frontiers in Psychology 2:395.

Seth AK, Friston KJ (2016) Active interoceptive inference and the emotional brain. Philosophical Transactions of the Royal Society B: Biological Sciences 371:20160007.

Shaffer F, Ginsberg JP (2017) An Overview of Heart Rate Variability Metrics and Norms. Frontiers in public health 5:258.

Smith R, Kuplicki R, Feinstein J, Forthman KL, Stewart JL, Paulus MP, Tulsa i, Khalsa SS (2020) A Bayesian computational model reveals a failure to adapt interoceptive precision estimates across depression, anxiety, eating, and substance use disorders. PLOS Computational Biology 16:e1008484.

Smith R, Feinstein JS, Kuplicki R, Forthman KL, Stewart JL, Paulus MP, Aupperle RL, Bodurka J, Savitz JB, Victor TA, Khalsa SS, Tulsa I (2021) Perceptual insensitivity to the modulation of interoceptive signals in depression, anxiety, and substance use disorders. Scientific Reports 11:2108. 
bioRxiv preprint doi: https://doi.org/10.1101/2021.12.06.471520; this version posted December 7, 2021. The copyright holder for this preprint (which was not certified by peer review) is the author/funder. All rights reserved. No reuse allowed without permission.

Southwick SM, Vythilingam M, Charney DS (2005) The psychobiology of depression and resilience to stress: implications for prevention and treatment. Annu Rev Clin Psychol 1:255-291.

Spielberger CD, Ritterband LM, Reheiser EC, Brunner TM (2003) The nature and measurement of depression. International Journal of Clinical and Health Psychology 3:209-234.

Sterling P (2012) Allostasis: A model of predictive regulation. Physiology \& Behavior 106:5-15.

Stuhrmann A, Suslow T, Dannlowski U (2011) Facial emotion processing in major depression: a systematic review of neuroimaging findings. Biology of Mood \& Anxiety Disorders 1:10.

Task Force of the European Society of Cardiology the North American Society of Pacing Electrophysiology (1996) Heart Rate Variability. Circulation 93:1043-1065.

Terhaar J, Viola FC, Bär K-J, Debener S (2012) Heartbeat evoked potentials mirror altered body perception in depressed patients. Clinical Neurophysiology 123:1950-1957.

Tracy LM, Gibson SJ, Georgiou-Karistianis N, Giummarra MJ (2017) Effects of explicit cueing and ambiguity on the anticipation and experience of a painful thermal stimulus. PLOS ONE 12:e0183650.

Vallat R (2018) Pingouin: statistics in Python. Journal of Open Source Software 3:1026.

van den Berg ME, Rijnbeek PR, Niemeijer MN, Hofman A, van Herpen G, Bots ML, Hillege H, Swenne CA, Eijgelsheim M, Stricker BH, Kors JA (2018) Normal Values of Corrected Heart-Rate Variability in 10Second Electrocardiograms for All Ages. Frontiers in Physiology 9:424.

Vaschillo EG, Bates ME, Vaschillo B, Lehrer P, Udo T, Mun EY, Ray S (2008) Heart rate variability response to alcohol, placebo, and emotional picture cue challenges: Effects of $0.1-\mathrm{Hz}$ stimulation. Psychophysiology 45:847-858.

Virtanen P et al. (2020) SciPy 1.0: fundamental algorithms for scientific computing in Python. Nature Methods 17:261-272.

Wiebking C, de Greck M, Duncan NW, Tempelmann C, Bajbouj M, Northoff G (2015) Interoception in insula subregions as a possible state marker for depression - an exploratory fMRI study investigating healthy, depressed and remitted participants. Frontiers in Behavioral Neuroscience 9:82.

Williams DP, Cash C, Rankin C, Bernardi A, Koenig J, Thayer JF (2015) Resting heart rate variability predicts selfreported difficulties in emotion regulation: a focus on different facets of emotion regulation. Frontiers in Psychology 6:261.

Zorzi L, Ranfaing S, Honoré J, Sequeira H (2021) Autonomic reactivity to emotion: A marker of sub-clinical anxiety and depression symptoms? Psychophysiology 58:e13774. 

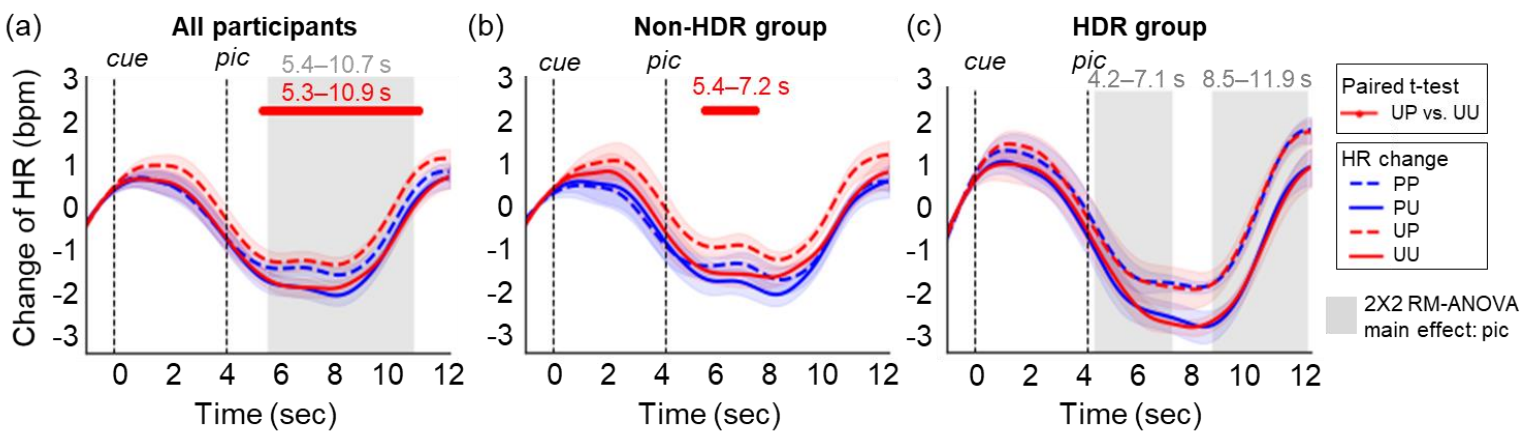

Figure 1 Time series of heart rate (HR) changes in four conditions, PP, PU, UP, and UU in (a) all participants, (b) the non-HDR group, and (c) the HDR group. The gray-shaded area indicates the time period showing the significant main effect of emotional picture in the cue $\times$ pic RM-ANOVA analysis and horizontal lines represent the period showing a significant difference in paired $t$-tests $\left(p_{\text {FDR-corrected }}<.05\right.$ and duration $>1 \mathrm{~s})$. The left and right vertical dashed lines indicate the onsets of cue and picture presentation, respectively. Red and blue shaded areas along the HR time series represent one standard error. HDR: high depressive risk; PP: predictable pleasant; PU: predictable unpleasant; UP: unpredictable pleasant; UU: unpredictable unpleasant. 
(a)

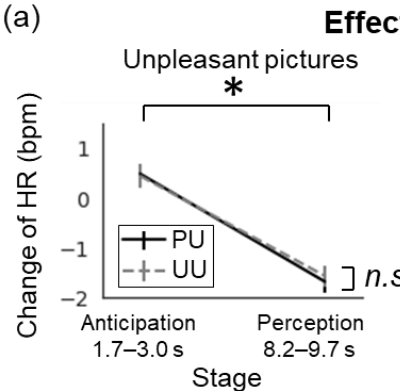

Effect of cue

Pleasant pictures

(b)

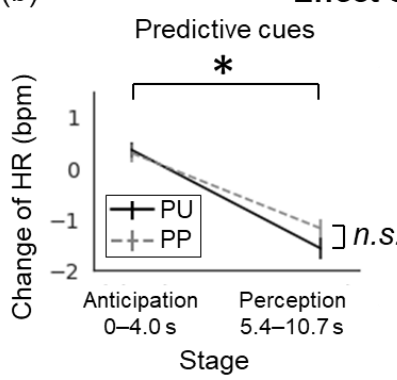

\section{Effect of picture}

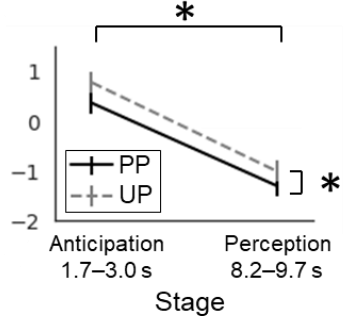

Unpredictive cues

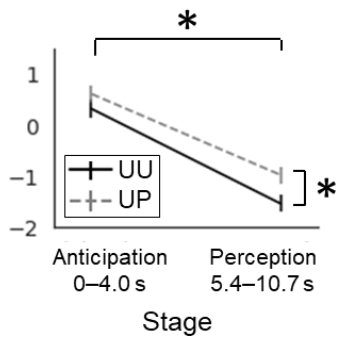

(c)

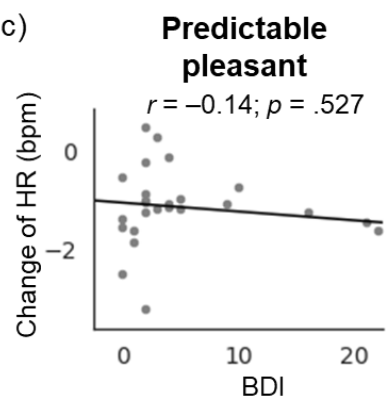

\section{Unpredictable} pleasant

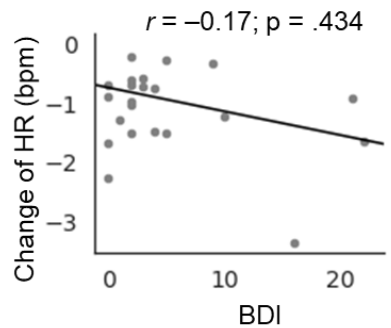

Predictable unpleasant

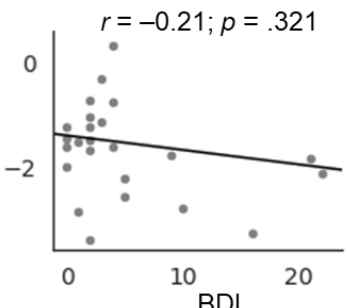

Unpredictable unpleasant $r=-0.61 ; \mathbf{p}=.001$ *

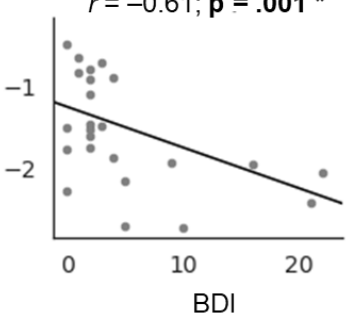

Figure 2 (a) Effect of cue and (b) effect of picture on changes in heart rate (HR). Error bars represent 1.96 within-subject standard errors and asterisks $\left({ }^{*}\right)$ denote the presence of significant main effects of stage (anticipation vs. perception) and condition (cues or valence) in $2 \times 2$ RM-ANOVA analysis $(p<.05)$. (c) Partial correlation ( $r$ ) between HR changes at perception stage (5.4-10.7 s) and level of HDR (BDI scores), controlled for age and gender. Asterisks $\left({ }^{\star}\right)$ denote the presence of a significant partial correlation $(p$ $<.05)$. Each circle denotes one participant and solid lines are linear fitted trend lines. HDR: high depressive risk; PP: predictable pleasant; PU: predictable unpleasant; UP: unpredictable pleasant; UU: unpredictable unpleasant; n.s.: not significant. 

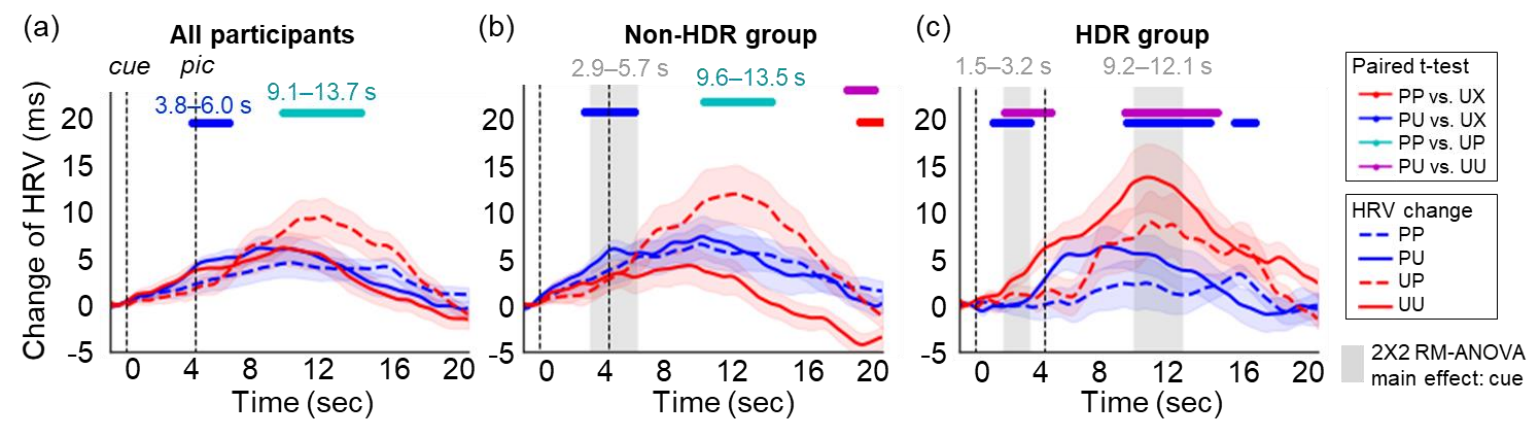

Figure 3 Time courses of heart rate variability (HRV) in the four conditions PP, PU, UP, and UU for (a) all participants, (b) non-HDR group, and (c) HDR group. The grey shaded area indicates the duration showing the significant main effect of cue in the cue x pic RM-ANOVA analysis and horizontal lines represent the duration showing a significant effect in paired $t$-tests $\left(p_{\text {uncorrected }}<.05\right.$ and duration $>1 \mathrm{~s}$ ). The left and right vertical dashed lines indicate the onsets of cue and picture presentation, respectively. Red and blue shaded areas along the HRV time series represent one standard error. HDR: high depressive risk; PP: predictable pleasant; PU: predictable unpleasant; UP: unpredictable pleasant; UU: unpredictable unpleasant; UX: unpredictable condition regardless of valence of pictures. 
(a)

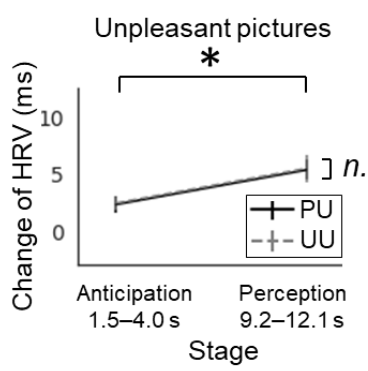

Effect of cue

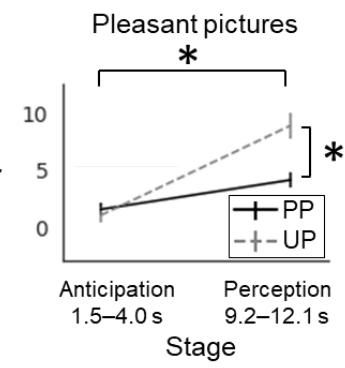

Effect of picture

(b)

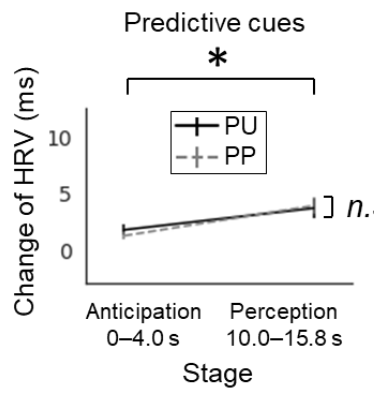

(c)

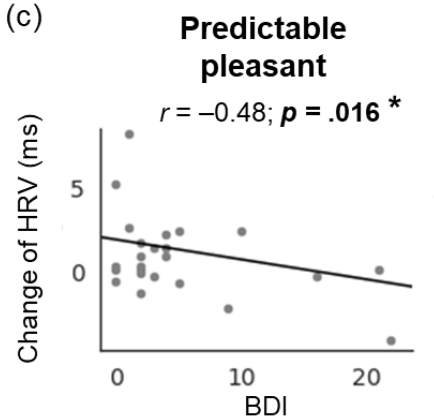

Unpredictable pleasant

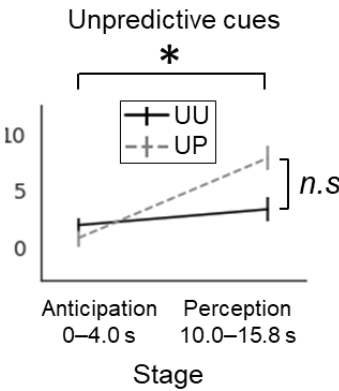

Predictable unpleasant $r=-0.53 ; p=.008$ *

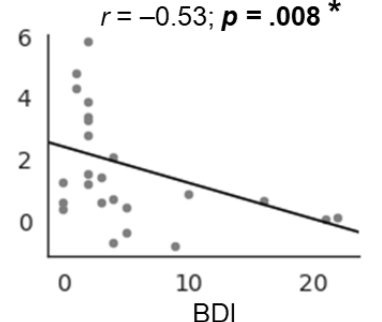

Unpredictable unpleasant

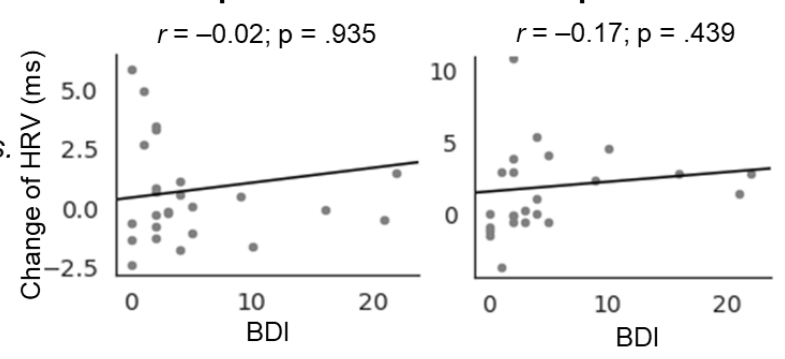

Figure 4 (a) Effects of cue and (b) of picture on changes on heart rate variability (HRV). Error bars represent 1.96 within-subject standard errors and asterisks $\left({ }^{*}\right)$ denote the presence of significant main effects of stage (anticipation vs. perception) and condition (cues or valence) in $2 \times 2$ RM-ANOVA analysis $(p<.05)$. (c) Partial correlation $(r)$ between HRV changes at anticipation stage $(1.5-4 \mathrm{~s})$ and level of HDR (BDI scores) controlled for age and gender. Asterisks $\left(^{*}\right)$ denote the presence of significant partial correlation $(p<.05)$. Each circle denotes one participant and solid lines are the linear fitted trend lines. PP: predictable pleasant; PU: predictable unpleasant; UP: unpredictable pleasant; UU: unpredictable unpleasant; n.s.: not significant. 


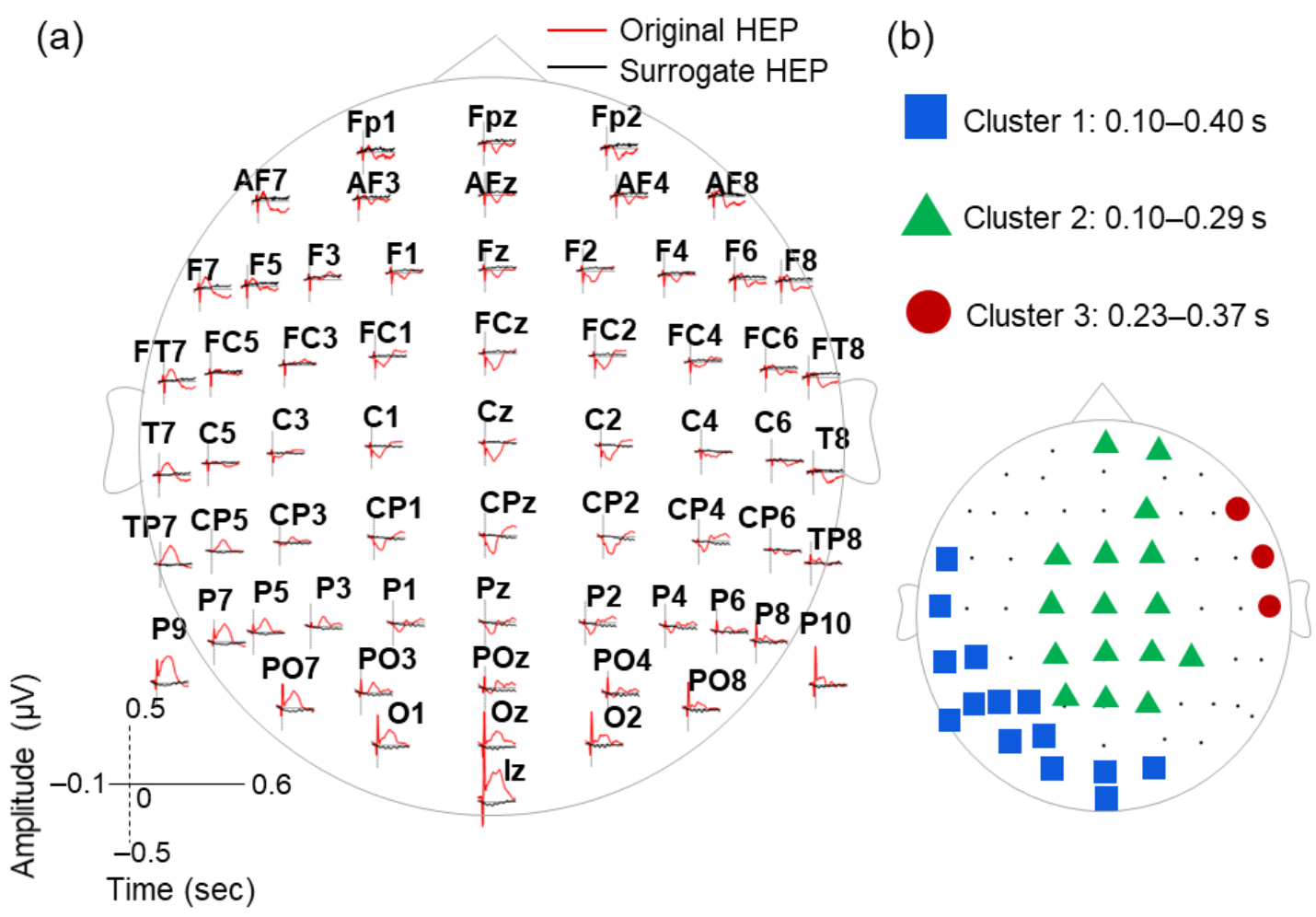

Figure 5 (a) Grand average of original heartbeat-evoked potential (HEP), obtained via epoching EEG signals based on actual R peaks, and surrogate HEP, obtained via epoching EEG signals based on randomly-shifted $R$ peaks, during -0.1 to $0.6 \mathrm{~s}$. (b) Blue squares, green triangles, and red circles indicate channel positions of HEP clusters 1, 2, and 3 determined by the spatiotemporal nonparametric clusterlevel test over the time series of HEP and surrogate HEP during 0.1-0.6 s. Significant durations for HEP clusters 1,2 , and 3 are $0.10-0.40$ s, $0.10-0.29$ s, $0.23-0.37$ s, respectively. 
(a)

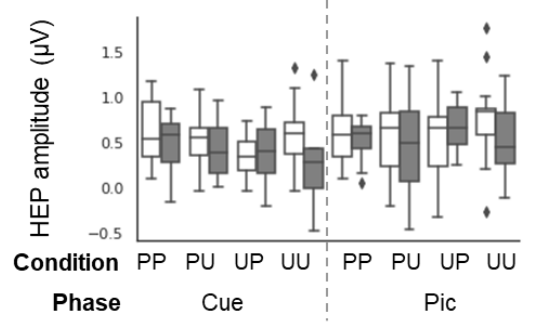

(b)

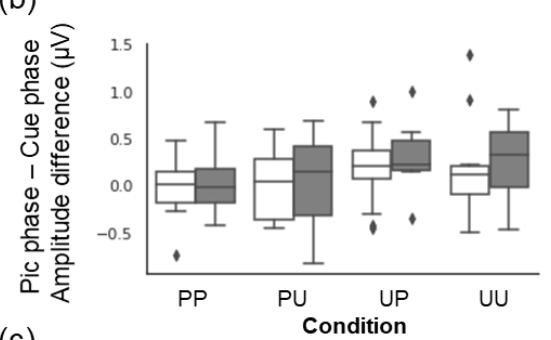

(c)

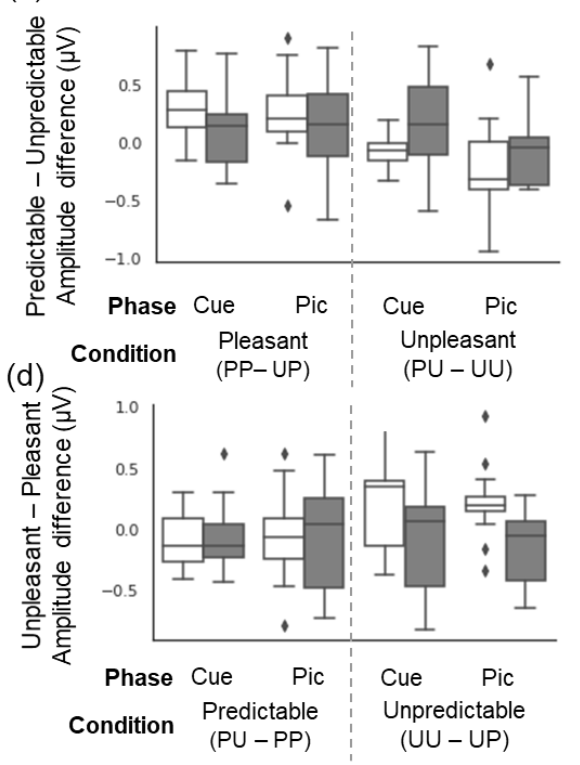

Cluster 2
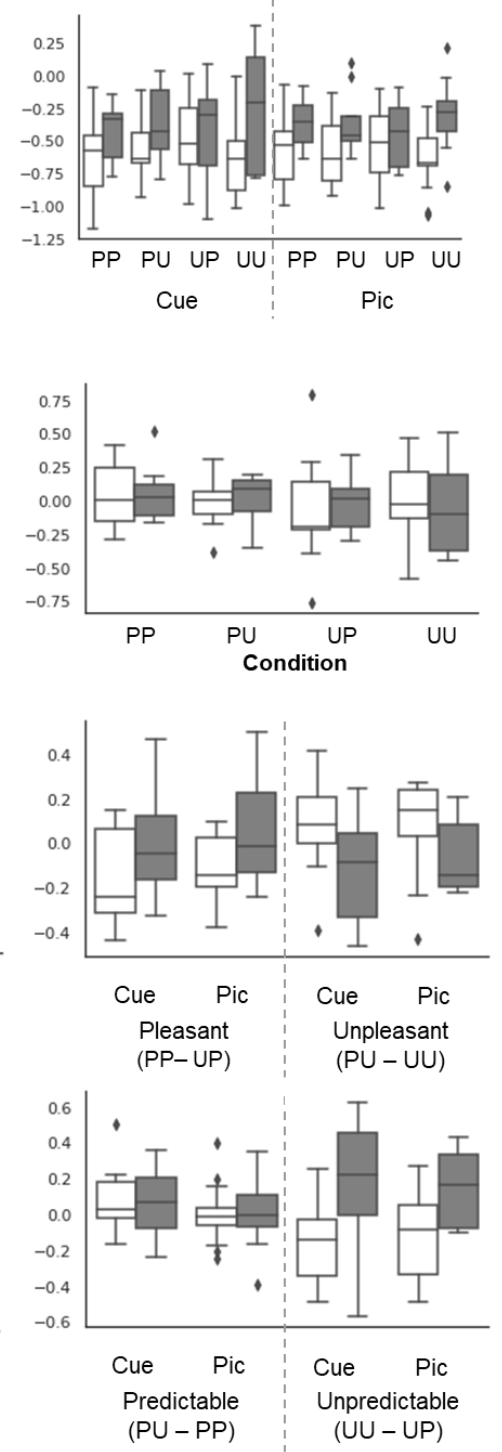

Cluster 3

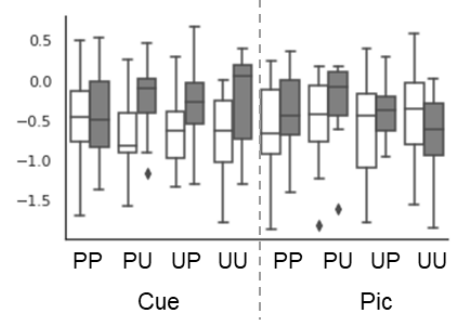

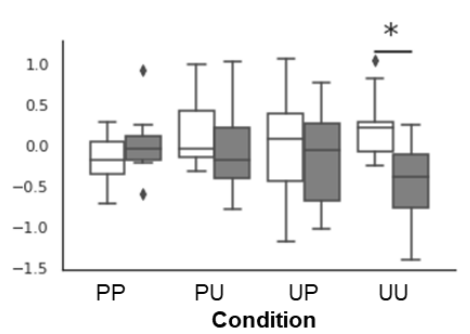
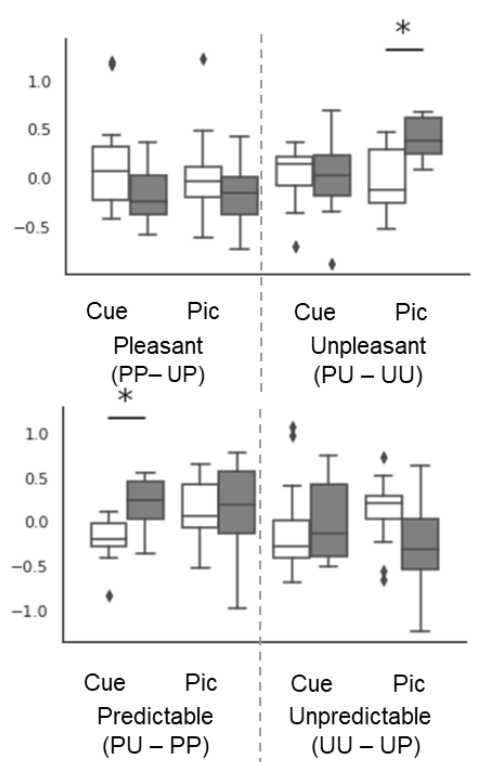

Figure 6 Boxplots of (a) heartbeat-evoked potential (HEP) amplitude for HEP clusters 1, 2, and 3 and amplitude difference for HEP clusters 1, 2, and 3 (b) between picture- and cue- phases (picture - cue) across all four conditions, (c) between predictable and unpredictable conditions, and (d) between pleasant and unpleasant conditions reflecting the effect of valence, reflecting the effect of certainty. White (left) and gray (right) boxes represent non-HDR and HDR groups, respectively. The horizontal bars connecting two bars indicate significantly different conditions between non-HDR and HDR groups in twosample $t$ tests. *: FDR-corrected $p<.05$. HDR: high depressive risk; PP: predictable pleasant; PU: predictable unpleasant; UP: unpredictable pleasant; UU: unpredictable unpleasant. 


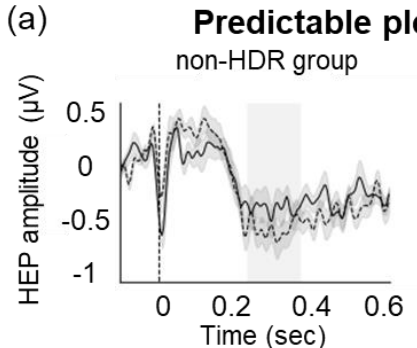

(c)

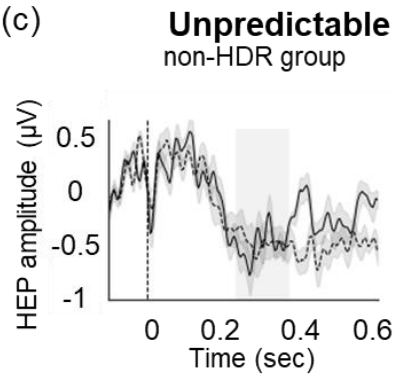

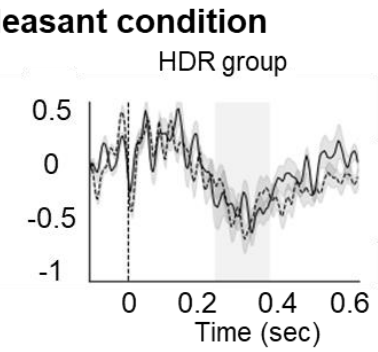

(b) Predictable unpleasant condition

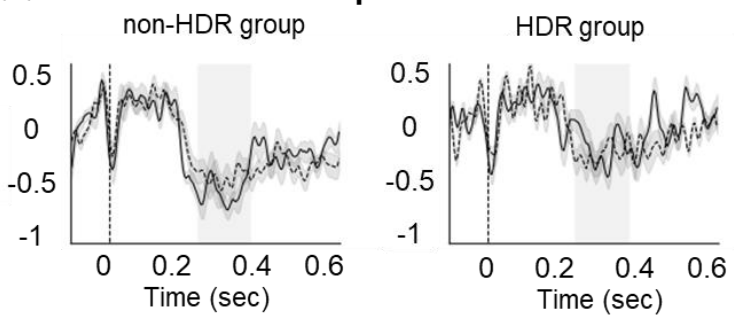

(d) Unpredictable unpleasant condition

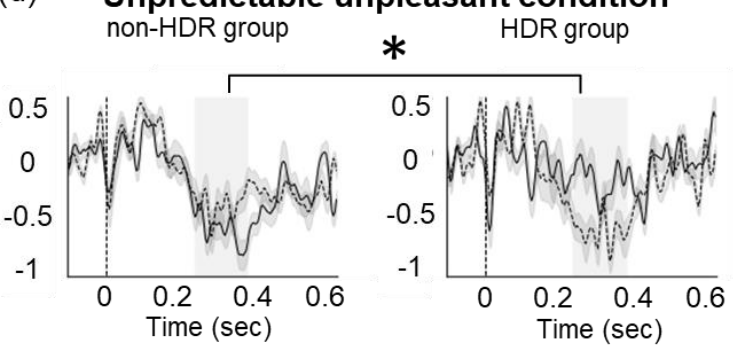

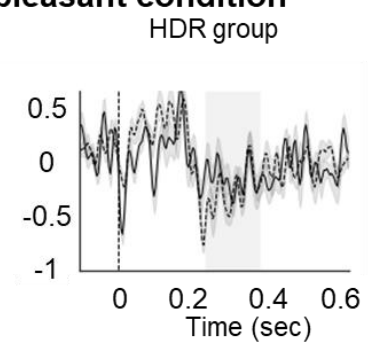

cue phase -.--. pic phase

Figure 7 Heartbeat-evoked potential (HEP) averaged over channels of the right frontotemporal cluster

(cluster 3) for (a) PP, (b) PU, (c) UP, and (d) UU conditions. Dashed lines represent the picture-phase and solid lines represent the cue-phase. The gray shaded area along HEP waveforms represents one standard error. Gray rectangles indicate a significantly different time-window, $0.23-0.37 \mathrm{~s}$, between the HEP and the surrogate HEP in the cluster-based spatiotemporal permutation test. The asterisk $\left({ }^{*}\right)$ indicates the significantly different effect of the HEP difference between the non-HDR and HDR groups (two-sample $t$ tests FDR-corrected $p<.05)$. HDR: high depressive risk; PP: predictable pleasant; PU: predictable unpleasant; UP: unpredictable pleasant; UU: unpredictable unpleasant. 
Predictable condition

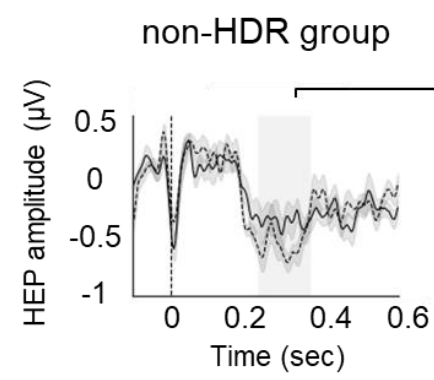

HDR group

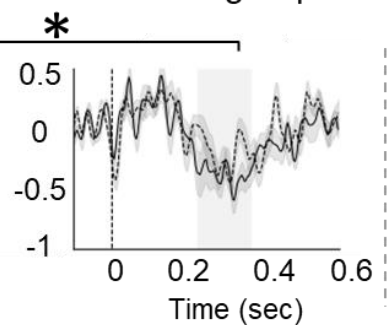

Unpredictable condition

non-HDR group

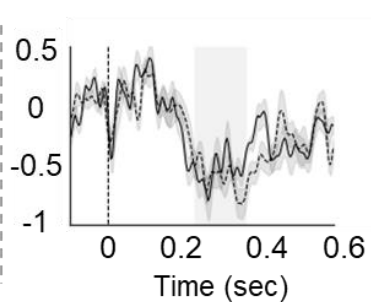

Time $(\mathrm{sec})$
HDR group

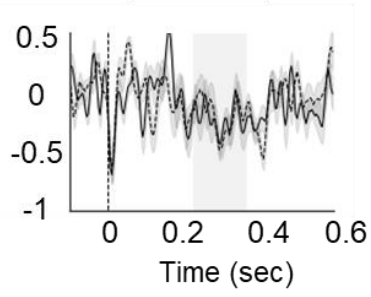

Figure 8 Grand averaged heartbeat-evoked potential (HEP) of the non-HDR and HDR groups plotted as a function of time for cluster 3 at cue phase. Dashed lines represent pleasant conditions and solid lines represent unpleasant conditions. The gray shaded area along the HEP waveform represents one standard error. The gray rectangle indicates the time period showing a significant difference between the grand averaged HEP and the surrogate HEP for cluster 3 in cluster-based spatiotemporal permutation test. The asterisk $(*)$ indicates that the difference of the valence induction effect of HEP between the non-HDR and HDR groups exceeded FDR-corrected $p<.05$ in two-sample $t$-tests, respectively. HDR: high depressive risk. 
(a)

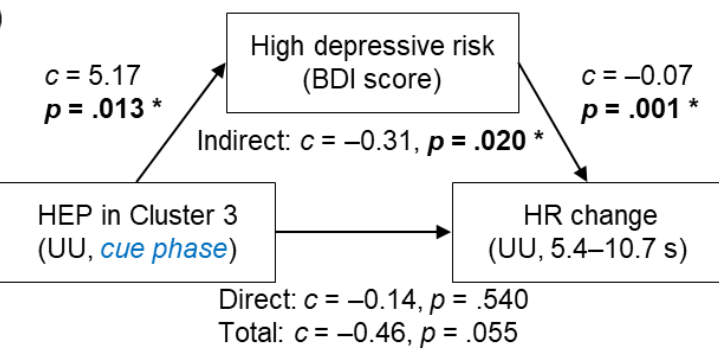

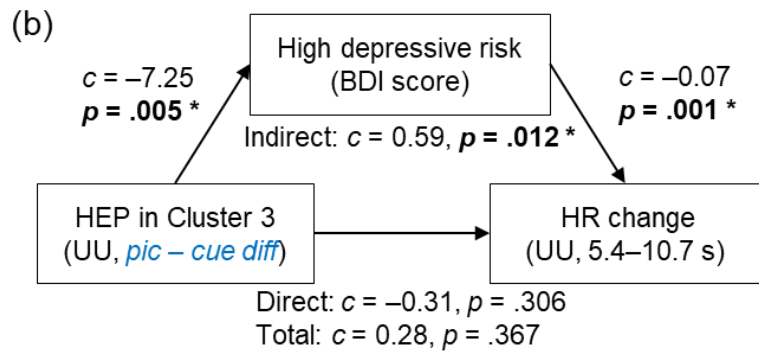

(b)
HR change

HEP in Cluster 3

Direct: $c=-0.31, p=.306$
Total: $c=0.28, p=.367$

Figure 9 Models to predict changes of heart rate (HR) under the unpredictable unpleasant (UU) condition

from (a) the heartbeat evoked potential (HEP) of cluster 3 at cue phase and (b) the difference of HEP amplitude between pic phase and cue phase (pic - cue diff) in cluster 3 mediated by HDR, which is quantified using a BDI score. ${ }^{*}: p<.05$. HDR: high depressive risk. 
Table 1 Comparison of the non-HDR and HDR groups and correlation with BDI for mean HR changes during 5.4-10.7 s. These $t$ values are results of two-sample $t$ tests. These $r$ values are partial correlation coefficients controlling for age and sex.

\begin{tabular}{|c|c|c|c|c|c|c|c|c|}
\hline \multirow{2}{*}{ Condition } & \multicolumn{2}{|c|}{ HR changes (bpm) } & \multicolumn{3}{|c|}{ Group comparison } & \multicolumn{3}{|c|}{ Correlation to BDI } \\
\hline & non-HDR group & HDR group & $t$ & $p$ & Hedges'g & $r$ & $p$ & $r^{2}$ \\
\hline PP & $-1.30(0.96)$ & $-1.30(0.40)$ & 0.02 & .983 & 0.01 & -.14 & .527 & .018 \\
\hline UP & $-0.86(0.76)$ & $-1.36(0.98)$ & 1.30 & .239 & 0.59 & -.17 & .434 & .028 \\
\hline$X P$ & $-1.08(0.68)$ & $-1.33(0.56)$ & 1.04 & .366 & 0.38 & -.19 & .379 & .035 \\
\hline $\mathrm{PU}$ & $-1.51(1.02)$ & $-2.22(0.70)$ & 1.93 & .075 & 0.75 & -.21 & .321 & .045 \\
\hline UU & $-1.28(0.52)$ & $-2.27(0.31)$ & 5.41 & $<.001 *$ & 2.08 & -.61 & $.001^{*}$ & .374 \\
\hline$X U$ & $-1.39(0.60)$ & $-2.24(0.40)$ & 3.86 & $<.001 *$ & 1.52 & -.43 & $.034^{*}$ & .188 \\
\hline$P P-P U$ & $0.21(1.58)$ & $0.91(1.01)$ & -1.23 & .229 & -0.48 & .08 & .707 & .007 \\
\hline$U P-U U$ & $0.42(0.63)$ & $0.92(1.15)$ & -1.12 & .293 & -0.55 & .43 & $.036^{*}$ & .185 \\
\hline$X P-X U$ & $0.32(0.82)$ & $0.91(1.77)$ & -1.75 & .096 & -0.74 & .28 & .192 & .076 \\
\hline
\end{tabular}

*: uncorrected $p<.05 ;$ mean $(S D)$

Table 2 Comparison of the non-HDR and HDR groups and correlation with BDI for mean HRV changes during 9.2-12.1 s. These $t$ values are results of two-sample $t$ tests. These $r$ values are partial correlation coefficients controlling for age and sex.

\begin{tabular}{|c|c|c|c|c|c|c|c|c|}
\hline \multirow[b]{2}{*}{ Condition } & \multicolumn{2}{|c|}{ HRV changes (ms) } & \multicolumn{3}{|c|}{ Group comparison } & \multicolumn{3}{|c|}{ Correlation to BDI } \\
\hline & $\begin{array}{c}\text { non-HDR } \\
\text { group }\end{array}$ & HDR group & $t$ & $p$ & Hedges'g & $r$ & $p$ & $r^{2}$ \\
\hline PP & $5.73(7.35)$ & $1.76(7.24)$ & 1.21 & .244 & 0.52 & -.34 & .104 & .116 \\
\hline UP & $11.24(11.72)$ & 8.10 (9.89) & 0.66 & .519 & 0.27 & -.31 & .148 & .093 \\
\hline$P P-U P$ & $-5.52(7.13)$ & $-6.34(11.79)$ & 0.18 & .861 & 0.09 & .08 & .703 & .007 \\
\hline PU & $6.44(8.60)$ & $4.75(6.17)$ & 0.52 & .607 & 0.21 & -.21 & .337 & .042 \\
\hline UU & $3.01(6.26)$ & $12.89(10.79)$ & -2.36 & $.040 *$ & -1.15 & .38 & .069 & .143 \\
\hline$P U-U U$ & $3.43(9.26)$ & $-8.14(6.14)$ & 3.44 & $.003 *$ & 1.35 & -.49 & $.016 *$ & .238 \\
\hline
\end{tabular}

*: uncorrected $p<.05 ;$ mean $(S D)$

Table 3 Correlation between the pic - cue difference of HEP amplitude and BDI scores for each cluster.

These $r$ values are partial correlation coefficients controlling for age and sex.

\begin{tabular}{|c|c|c|c|c|c|c|c|c|c|}
\hline \multirow{2}{*}{ Condition } & \multicolumn{3}{|c|}{ HEP cluster 1} & \multicolumn{3}{|c|}{ HEP cluster 2} & \multicolumn{3}{|c|}{ HEP cluster 3} \\
\hline & $r$ & $p$ & $r^{2}$ & $r$ & $p$ & $r^{2}$ & $r$ & $p$ & $r^{2}$ \\
\hline $\mathrm{PP}$ & -.04 & .839 & .002 & .07 & .746 & .005 & .24 & .264 & .056 \\
\hline PU & -.10 & .656 & .009 & .03 & .889 & .001 & .01 & .955 & .000 \\
\hline UP & .06 & .781 & .004 & .14 & .516 & .019 & -.13 & .548 & .017 \\
\hline UU & .30 & .156 & .089 & -.12 & .576 & .014 & -.56 & $.005^{*}$ & .308 \\
\hline
\end{tabular}

*: uncorrected $p<.01$ 
Table 4 Correlation between amplitude of the HEP and HR changes during 5.4-10.7 s for each cluster.

These $r$ values are partial correlation coefficients controlling for age and sex.

\begin{tabular}{|c|c|c|c|c|c|c|c|c|c|c|}
\hline \multirow{2}{*}{$\begin{array}{l}\text { HEP } \\
\text { phase }\end{array}$} & \multirow{2}{*}{ Condition } & \multicolumn{3}{|c|}{ HEP cluster 1} & \multicolumn{3}{|c|}{ HEP cluster 2} & \multicolumn{3}{|c|}{ HEP cluster 3} \\
\hline & & $r$ & $p$ & $r^{2}$ & $r$ & $p$ & $r^{2}$ & $r$ & $p$ & $r^{2}$ \\
\hline \multirow{4}{*}{ cue } & PP & -.21 & .321 & .045 & -.03 & .900 & .001 & .07 & .739 & .005 \\
\hline & PU & .44 & $.033^{*}$ & .190 & -.01 & .965 & .000 & -.27 & .208 & .071 \\
\hline & UP & .11 & .602 & .013 & -.01 & .563 & .015 & -.23 & .282 & .052 \\
\hline & UU & .34 & .107 & .114 & -.38 & .069 & .143 & -.40 & .055 & .158 \\
\hline \multirow{4}{*}{ pic } & PP & -.40 & .055 & .158 & .09 & .673 & .008 & .11 & .611 & .012 \\
\hline & PU & .21 & .333 & .043 & -.03 & .888 & .001 & -.37 & .079 & .133 \\
\hline & UP & .36 & .085 & .129 & -.32 & .126 & .103 & -.09 & .677 & .008 \\
\hline & UU & .44 & $.030 *$ & .197 & -.55 & $.005^{*}$ & .305 & -.27 & 210 & .070 \\
\hline
\end{tabular}

*: uncorrected $p<.05$ 